LMU-ASC 34/10

\title{
On the Heterotic World-sheet Instanton Superpotential and its individual Contributions
}

\author{
Gottfried Curio ${ }^{1,2}$ \\ Arnold-Sommerfeld-Center for Theoretical Physics \\ Department für Physik, Ludwig-Maximilians-Universität München \\ Theresienstr. 37, 80333 München, Germany
}

\begin{abstract}
For supersymmetric heterotic string compactifications on a Calabi-Yau threefold $X$ endowed with a vector bundle $V$ the world-sheet superpotential $W$ is a sum of contributions from isolated rational curves $\mathcal{C}$ in $X$; the individual contribution is given by an exponential in the Kähler class of the curve times a prefactor given essentially by the Pfaffian which depends on the moduli of $V$ and the complex structure moduli of $X$. Solutions of $D W=0$ (or even of $D W=W=0$ ) can arise either by nontrivial cancellations between the individual terms in the summation over all contributing curves or because each of these terms is zero already individually. Concerning the latter case conditions on the moduli making a single Pfaffian vanish (for special moduli values) have been investigated. However, even if corresponding moduli - fulfilling these constraints - for the individual contribution of one curve are known it is not at all clear whether one choice of moduli exists which fulfills the corresponding constraints for all contributing curves simultaneously. Clearly this will in general happen only if the conditions on the 'individual zeroes' had already a conceptual origin which allows them to fit together consistently. We show that this happens for a class of cases. In the special case of spectral cover bundles we show that a relevant solution set has an interesting location in moduli space and is related to transitions which change the generation number.
\end{abstract}

\footnotetext{
${ }^{1}$ gottfried.curio@physik.uni-muenchen.de

${ }^{2}$ supported by DFG grant CU 191/1-1
} 


\section{Introduction}

For a heterotic string compactification on a Calabi-Yau threefold $X$ endowed with a stable holomorphic vector bundle $V$ the world-sheet instanton superpotential $W$ is a sum of contributions from isolated rational curves $\mathcal{C}$ (assumed to be smooth) in $X$

$$
W=\sum_{\mathcal{C}} W_{\mathcal{C}}
$$

(we call such curves 'instanton curves'). The individual contribution is given by

$$
W_{\mathcal{C}}=\frac{P f a f f_{\mathcal{C}}(v, z)}{D_{\mathcal{C}}(z)^{2}} e^{i C \cdot J}
$$

where the Pfaffian depends on the vector bundle moduli of $V$ and the complex structure moduli of $X$ (collectively denoted by $v$ and $z$, respectively), the denominator $D$ depends on $z$, and the exponential term gives the dependence on the Kähler class $J=k_{i} J_{i}$ (we denote the Kahler moduli collectively by $k$ and the homology class of $\mathcal{C}$ by ${ }^{3} C$ where $\left.C \cdot J=\int_{\mathcal{C}} J\right)$. Actually Pfaff$f_{\mathcal{C}}(v, z)=\operatorname{Pfaff}\left(\bar{\partial}_{V(-1)}(\mathcal{C})\right)$ and $D_{\mathcal{C}}(z)=\operatorname{det} \bar{\partial}_{\mathcal{O}(-1)}(\mathcal{C})$ times the constant $\operatorname{det}^{\prime} \bar{\partial}_{\mathcal{O}}$ (here one considers twists by $\mathcal{O}(-1)$ on the rational curve $\mathcal{C}$ ).

As the Kähler factor depends only on the homology class $C=[\mathcal{C}]$ of $\mathcal{C}$ one can write

$$
W=\sum_{C}\left(\sum_{[\mathcal{C}]=C} \frac{P f a f f_{\mathcal{C}}(v, z)}{D_{\mathcal{C}}(z)^{2}}\right) e^{i C \cdot J}=: \sum_{C} P_{C} e^{i C \cdot J}
$$

remarks: 1) Actually, because of the phase-factor subtlety [1], the summation in (1.3) is conceptually more difficult; in $J=B+i \tilde{J}$ the $B$-field contribution has strictly speaking to be considered together with the Pfaffian factor. Connected with this is that the naive moduli space factorisation $\mathcal{M} \times \mathcal{M}_{K}=\mathcal{M}_{V} \times \mathcal{M}_{c x} \times \mathcal{M}_{K}$ does not hold: clearly because of the holomorphy and stability condition for $V$ its moduli space $\mathcal{M}_{V}$ is actually fibered over $\mathcal{M}_{c x} \times \mathcal{M}_{K}$; but the phase-factor subtlety for the $B$-field is related to the anomaly cancellation equation which connects its field strength $H$ to the quantity $\operatorname{tr} F \wedge F$ which depends in turn on $\mathcal{M}_{V}$. We will be able to ignore these issues in this paper. We will write the naive product, and, when speaking of the full $\mathcal{M}_{c x}$ or $\mathcal{M}_{K}$ as possible moduli sets for $v \in \mathcal{M}_{V}$ in a solution, we mean the regions where $V_{v}$ is holomorphic or stable.

2) Another subtlety is the fact that the existence of holomorphic rational curves $\mathcal{C}$ depends on the complex structure moduli of $X$; however, if $\mathcal{C}$ is isolated and even already rigid infinitesimally (actually even this is necessary for contribution) then the normal bundle of $\mathcal{C}$ is $\mathcal{O}(-1) \oplus \mathcal{O}(-1)$ and $\mathcal{C}$ deforms with $X$ in all directions in $\mathcal{M}_{c x}(X)$; so such contributing curves do not arise newly by specialisation.

To place the following considerations in their proper context we distinguish first a number of different but closely related questions pertaining to $W$ and the $W_{\mathcal{C}}$.

\footnotetext{
${ }^{3}$ this is the notation in this section; in the rest of the paper $C$ denotes various spectral cover objects
} 


\subsection{Direct investigation of the full superpotential $W$}

Here and in the following subsection we will distinguish in each case considerations from the point of view of conformal invariance of the sigma model, related to universal (in the moduli) vanishing results, from considerations under the guiding philosophy to use actually a superpotential (which does not vanish identically) to fix the moduli.

\subsubsection{The universal case}

The most immediate question, and the one which was originally first investigated, when the world-sheet point of view prevailed, was naturally the following (the term 'universal' refers to $W$ being identically zero, independent of any special choice of moduli values):

\section{Question W(universal)}

For which pairs $(X, V)$ is $W \equiv 0$ ?

If this holds for a pair $(X, V)$ then one must actually have a seemingly even stronger result (but which is really equivalent as being enforced by $W \equiv 0$ ): as $W=0$ should hold independently of any choice of specific values for the Kahler moduli, terms with Pfaffians for curves in different homology classes cannot cancel against each other; that is, each Kahler prefactor $P_{C}$ has to vanish individually. This gives the equivalent

\section{$\underline{\text { Question W(universal) }}{ }^{\prime}$}

For which pairs $(X, V)$ is $P_{C}=\sum_{[\mathcal{C}]=C} \frac{P f a f f_{\mathcal{C}}(v, z)}{D_{\mathcal{C}}(z)^{2}} \equiv 0$ for all homology classes $C$ ?

Here one has the remarkable result [3] (assuming $c_{2}(V)=c_{2}(X)$ ) that this holds via a nontrivial cancellation between nonzero terms for example for hypersurfaces or complete intersections $X$ in an ambient (weighted) projective space $Y$, with $V$ arising as restriction from a bundle on $Y$ (for a similar simpler case cf. [4]). Other cases, where this holds not from a nontrivial cancellation between nonzero terms, but because of vanishing of each

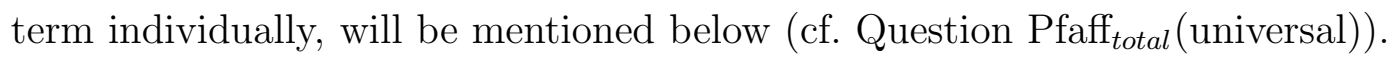

\subsubsection{The special case}

This first question above, the search for $W \equiv 0$, reflects directly the stringy or worldsheet point of view and was much discussed in the late 80's. In the point of view which looks on an effective low energy action in four dimensions, which prevailed in the mid-90's and later, issues like moduli stabilisation from such superpotentials were the prominent focus. Here the question is this: 


\section{Question W(special)}

For which points $(t, k) \in \mathcal{M} \times \mathcal{M}_{K}$ is $W(t, k)=0$ ?

A refinement of this question, relevant for solutions of the system $D W=W=0$, is: Question W(special $)^{\geq 2}$

For which points $t \in \mathcal{M}$ is $W(t, k)=0$ to second or higher order ?

Properly speaking, of course, instead of asking first where $W$ vanishes and then where it does so even to higher order, the first physical question (which is here that of moduli stabilisation by demanding the supersymmetry conditions) amounts to where in moduli space $D W(t, k)=0$ is solved; only then one evaluates the negative or zero tree-level cosmological constant $V=-3|W|^{2} e^{K}$ and may ask for Minkowski vacua which have also $W(t, k)=0$ (which reduces then, when posed as an additional condition, the covariant derivatives to ordinary ones, and leads one to pose the question above).

\subsection{Investigation of $W$ from the perspective of its individual contributing summands from isolated rational curves $\mathcal{C}$}

The questions above represent one line of reasoning where one takes into consideration from the outset the total world-sheet superpotential $W$, with all individual contributions summed up. But as $W$ is a rather complex quantity one also investigates, in a second line of investigation, first its individual contributions from the appropriate rational curves $\mathcal{C}$.

\subsubsection{The universal case}

This leads to the following question (specific in $\mathcal{C}$, but universal in the identical vanishing): Question Pfaff (universal)

For which pairs $(X, V)$ and isolated (smooth) rational curves $\mathcal{C}$ is $\operatorname{Pfaf} f_{\mathcal{C}} \equiv 0$ ?

Here one has the criterion $^{4}[1]$ that this happens just if $V$, which restricts to $\mathcal{C}$ as a direct sum of line bundles, is nontrivial, i.e. $\neq \oplus \mathcal{O}_{\mathcal{C}}(0)$.

In view of our goal to understand the full $W$ one is led naturally to the question:

\section{Question Pfaff total $_{\text {(universal) }}$}

For which pairs $(X, V)$ is $\operatorname{Pfaf} f_{\mathcal{C}} \equiv 0$ for all isolated (smooth) rational curves $\mathcal{C}$ ?

One case is the tangent bundle $V=T X$ as $\left.V\right|_{\mathcal{C}}=\mathcal{O}(2) \oplus \mathcal{O}(a) \oplus \mathcal{O}(-a-2)$ is always nontrivial. For $X$ the generic quintic in $\mathbf{P}^{4}$ a stable deformation $V$ of $T X \oplus \mathcal{O}$ exists having the required property for all lines [5]. For yet another case cf. [6].

\footnotetext{
${ }^{4}$ the analysis is done for $S O(32)$ bundles or $E_{8} \times E_{8}$ bundles with structure group in $S O(16) \times S O(16)$
} 


\subsubsection{The special case}

Now let us change again the perspective from results holding universally in the moduli to the philosophy where special moduli are chosen from the conditions imposed. So one now asks, in case that $P f a f f_{\mathcal{C}}$ does not vanish identically, again the specified question:

\section{Question Pfaff ${ }_{\mathcal{C}}$ (special)}

For which points $t \in \mathcal{M}$ is $\operatorname{Pfaf} f_{\mathcal{C}}(t)=0$ ?

Although one has the mentioned criterion of nontriviality of $\left.V\right|_{\mathcal{C}}$, to detect, whether this actually holds or not in dependence on the moduli, is still a nontrivial task.

Again of interest, for finding supersymmetric solutions (which are in this approach necessarily Minkowski vacua), is also the refinement:

\section{Question Pfaff ${ }_{\mathcal{C}}$ (special) $)^{\geq 2}$}

For which points $t \in \mathcal{M}$ is $\operatorname{Pfaf} f_{\mathcal{C}}(t)=0$ to second or higher order ?

Some examples, concerning the last two questions, were computed in a computer calculation in [7] and understood more conceptually in [10, 11].

As in the universal case, in view of the fact that the total contribution in $W$ is a sum over all contributions from the individual curves $\mathcal{C}$, the following question is now of importance and the one we focus on in the present paper:

\section{Question Pfaff total $_{\text {(special) }}$}

For which points $t \in \mathcal{M}$ is $\operatorname{Pfa} f f_{\mathcal{C}}(t)=0$ for all instanton curves $\mathcal{C}$ simultaneously ?

To use this approach to find supersymmetric (Minkowski) vacua one needs control also over the derivatives and so one is led again to the refinement

\section{Question Pfaff total $_{\text {(special })^{\geq 2}}$}

For which points $t \in \mathcal{M}$ is $\operatorname{Pfaf} f_{\mathcal{C}}(t)=0$ to second or higher order for all instanton curves $\mathcal{C}$ simultaneously ?

By posing theses questions we have replaced the original system of conditions

$$
\partial_{v_{l}} W=\partial_{z_{m}} W=\partial_{k_{j}} W=W=0, \quad \forall l, \forall m, \forall j
$$

for the total superpotential by the system of equations pertaining to the instanton contributions $P$ faf $f_{\mathcal{C}_{i}}$ from all the individual instanton curves $\mathcal{C}_{i}$

$$
\partial_{v_{l}} P f a f f_{\mathcal{C}_{i}}=\partial_{z_{m}} P f a f f_{\mathcal{C}_{i}}=P f a f f_{\mathcal{C}_{i}}=0 \quad \forall l, \forall m \text { and } \forall i
$$

(with $v_{k}, z_{j}$ and $k_{m}$ local coordinates on $\mathcal{M}_{V}, \mathcal{M}_{c x}$ and $\mathcal{M}_{K}$, resp.). We will collect some general remarks about the relation of these systems in sect. 1.3 and especially in sect. 2 below where we treat the moduli freedom in $\mathcal{M}_{c x}$ and $\mathcal{M}_{K}$ for solutions of (1.5) in detail. 


\subsection{Relation of these two lines of consideration}

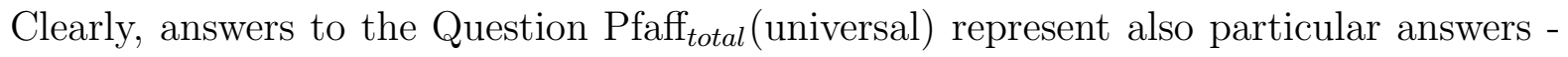
though one might say the trivial ones (no cancellation between nonzero summands) - to the Question W(universal); similarly answers to the Question Pfaff total $_{\text {(special) represent }}$ also particular answers - though, again, one might say the trivial ones (no cancellation) to the Question W(special) above (and similarly if one takes into account higher orders).

These assessments reflect the perspective looking from cases with an actual cancellation between summands. However one can see the situation also from the following angle: let us assume we have investigated some individual Pfaffians $\operatorname{Pfaf} f_{\mathcal{C}_{i}}(v, z)$ and found, for each $i$ individually, some solution sets, i.e. $(v, z) \in \mathcal{S}_{i} \subset \mathcal{M} \Longrightarrow \operatorname{Pfaff}_{\mathcal{C}_{i}}(v, z)=0$ (the issue of higher multiplicities of the zeroes may be included in the discussion).

Then it is a priori quite surprising that a moduli choice which makes the Pfaffian for $\mathcal{C}_{1}$ vanish should also do so for $\mathcal{C}_{2}$, let alone for all $\mathcal{C}_{i}$. Although it is true that the question of vanishing for $P f a f f_{\mathcal{C}_{i}}$ usually does not see all the moduli of $V$ (as some sort of restriction to the part of the set-up relevant to $\mathcal{C}_{i}$ is involved; cf. below) and therefore even a sharp (narrow) condition on the (restricted set of) moduli relevant to $\mathcal{C}_{i}$ enlarges to a more relaxed condition when considered for the full set of moduli of $V$, it nevertheless should usually lead to a contradictory system of conditions when posed for all $\mathcal{C}_{i}$ at once.

So, from this perspective, the possibility of getting zeroes of $W$ from getting individual zeroes for all the $\operatorname{Pfaf} f_{\mathcal{C}_{i}}$ (of suitable order) looks almost as mysterious as a potential cancellation of individual nonzero summands: here again some sort of hidden cooperation must happen to solve this now (usually) highly overdetermined system (cf. sect. 2.3).

\subsection{Plan of the paper}

After some preliminary remarks in sect. 2 we start our investigation in sect. 3 in a set-up where results about the vanishing behaviour of individual Pfaffians were found: the case of spectral cover bundles over an elliptic Calabi-Yau space $\pi: X \rightarrow B$. Here we will be able to achieve a definitive answer to our main question(s) mentioned in subsection 1.2.2. However, our result will suffer from a serious drawback: in this approach one has control over the vanishing behaviour of Pfaffians only for base instanton curves $\mathcal{C}_{i} \subset B$.

Therefore we make a second start in sect. 4 where we define a new class of bundles with the leading idea that the role played by the base $B$ in the spectral construction is now played by the full (arbitrary) Calabi-Yau space $X$ itself. For these bundles we succeed in giving a positive answer now for all instanton curves. In sect. 5 we conclude. 


\section{Some preliminary remarks}

After having noted already in sect. 1.3 that our general system of conditions (where we use the notation $C_{i}=\left[\mathcal{C}_{i}\right]$ for the homology class, $D_{i}:=D_{\mathcal{C}_{i}}$ and $k \cdot C_{i}=\sum k_{j} n_{j}^{(i)}$ )

$$
\begin{aligned}
W & =\sum_{\mathcal{C}_{i}} \operatorname{Pfaff}_{\mathcal{C}_{i}} D_{i}^{-2} e^{i k \cdot C_{i}}=0 \\
\partial_{k_{j}} W & =\sum_{\mathcal{C}_{i}} \operatorname{Pfaff}_{\mathcal{C}_{i}} D_{i}^{-2} i n_{j}^{(i)} e^{i k \cdot C_{i}}=0 \\
\partial_{z_{m}} W & =\sum_{\mathcal{C}_{i}}\left[\left(\partial_{z_{m}} \operatorname{Pfaff} f_{\mathcal{C}_{i}}\right) D_{i}^{-2}+\operatorname{Pfaff} f_{\mathcal{C}_{i}} \partial_{z_{m}} D_{i}^{-2}\right] e^{i k \cdot C_{i}}=0 \\
\partial_{v_{l}} W & =\sum_{\mathcal{C}_{i}}\left(\partial_{v_{l}} \operatorname{Pfaff} f_{\mathcal{C}_{i}}\right) D_{i}^{-2} e^{i k \cdot C_{i}}=0
\end{aligned}
$$

(for brevity of notation we suppressed the specific solution points in $\mathcal{M}_{V} \times \mathcal{M}_{c x} \times \mathcal{M}_{K}$ where all the expressions are actually evaluated to give zero; similarly below) admits as particular solutions the solutions of the other system (called the 'special solutions' below)

$$
\begin{aligned}
\operatorname{Pfaff} f_{\mathcal{C}_{i}} & =0 \\
\partial_{z_{m}} P f a f f_{\mathcal{C}_{i}} & =0 \\
\partial_{v_{l}} P f a f f_{\mathcal{C}_{i}} & =0
\end{aligned}
$$

we want to collect in this short section some (formal) notes on the possible freedom in $\mathcal{M}_{c x} \times \mathcal{M}_{K}$ for solutions characterising (or not, cf. below) such more special solutions.

\subsection{Remarks on the moduli freedom in $\mathcal{M}_{c x} \times \mathcal{M}_{K}$ for our special solutions}

We begin with the 'reduction' from $W$ to the $P_{C}=\sum_{\left[\mathcal{C}_{i}\right]=C} \operatorname{Pfaf} f_{\mathcal{C}_{i}} D_{i}^{-2}$; the possibility of a second 'reduction', from the $P_{C}$ to the $\operatorname{Pfaf} f_{\mathcal{C}_{i}}$, will be discussed in sect.2.2 below. Remarks: a) Note that for 'special solutions' (which fix the moduli $(v, z)$ to lie in a certain region $S_{\mathcal{M}}$ in $\mathcal{M}=\mathcal{M}_{V} \times \mathcal{M}_{c x}$ ) the Kahler moduli remain completely flat directions. Actually this will be the case for any solution which makes all the individual $P_{C}$ vanish (for certain values in $\mathcal{M}$ ). One has also the converse, i.e. the precise association

\section{$\underline{\text { Solutions }}$}

with $\left\{\begin{array}{c}(v, z) \text { in a certain } \\ \text { region in } \mathcal{M}_{V} \times \mathcal{M}_{c x}\end{array}\right\}$

times \{flat Kähler directions\}

\section{$\underline{\text { Solutions }}$}

where for $\left\{\begin{array}{c}(v, z) \text { in a certain } \\ \text { region in } \mathcal{M}_{V} \times \mathcal{M}_{c x}\end{array}\right\}$

one has $\left\{P_{C}=\partial_{z_{m}} P_{C}=\partial_{v_{l}} P_{C}=0, \forall C\right\}$ 
b) Recall that the conditions $\partial_{i} W=0=W$ for a supersymmetric Minkowski vacuum are $h_{m}+1$ conditions for $h_{m}$ variables where $h_{m}=h_{K}+h_{c x}+h_{V}$ is the number of moduli, which comprises $h_{K}=h^{1,1}(X)$ Kähler moduli, $k_{c x}=h^{2,1}(X)$ complex structure moduli and $h_{V}=h^{1}(X, E n d V)$ vector bundle moduli. So, without the Minkowski condition this system of $h_{m}$ equations (then of course with the covariant derivatives $D_{i} W=0$ ) for $h_{m}$ complex variables should have generically a solution. That a corresponding supersymmetric vacuum should be a Minkowski vacuum makes the question nontrivial.

If, however, one of the equations $\partial_{i} W=0$ would not pose effectively a condition because one has $\partial_{i} W \equiv 0$, i.e. the derivative vanishes identically (the modulus labeled by $i$ being a flat direction), then one would be back to the generic counting argument, now even including the additional Minkowski condition $W=0$. So one has the following

\section{$\underline{\text { Fact }}$}

If the moduli space of a pair $(X, V)$ contains a flat direction one will generically have a supersymmetric Minkowski vacuum.

Now, in our context the following distinction becomes important which will be related to the existence of such flat directions in the Kähler moduli part of $\mathcal{M} \times \mathcal{M}_{K}$.

\section{Definition 'Generically Kähler-determined Bundles'}

A pair $(X, V)$ is called 'generically Kähler-determined' if the real span (of the homology classes) of its contributing $\left(P_{C} \not \equiv 0\right)$ instanton curves generates $H_{2}(X, \mathbf{R})$.

Note that one is speaking here of contributing classes $C$ of instanton curves $\mathcal{C}$. If $[\mathcal{C}]=$ $\sum_{j=1}^{h_{K}} n_{j} C_{j}$ is a decomposition of an instanton curve in a homology basis $\left(n_{j} \in \mathbf{Z}\right.$; the $C_{j}$ are in general not classes of instanton curves) and $J=\sum_{m=1}^{h_{K}} k_{m} J_{m}$ a dual decomposition of a general Kähler class $\left(k_{m}\right.$ are the Kähler moduli) such that $\mathcal{C} \cdot J=\sum n_{j} k_{j}$ one has

$$
\partial_{k_{m}} W=\sum P_{C} i n_{m} e^{i \mathcal{C} \cdot J}
$$

If $V$ over $X$ is not generically Kähler-determined the span of contributing instanton classes will not contain, say, $C_{h_{K}}$ : all contributing $C$ will have $n_{h_{K}}=0$ so that $\partial_{k_{h_{K}}} W \equiv 0$.

\section{$\underline{\text { Fact }}$}

A bundle $V$ over $X$ which is not generically Kähler-determined has a flat direction.

So bundles which are not generically Kähler-determined will generically have a supersymmetric Minkowski vacuum. But usually interesting bundles will be generically Kähler-determined such that, despite the generic counting argument against a supersymmetric Minkowski vacuum, the question arises for suitable special moduli choices providing such a vacuum (cf. also sect. 3.6). As remark a) above shows the moduli choices in our solutions will nevertheless lead to completely flat Kähler directions. 
c) After the Kähler moduli, which we considered more closely in remarks a) and b), let us also have a closer look on the complex structure moduli.

We start with a general remark. One may sometimes encounter special situations where each Pfaffian $\operatorname{Pfaf} f_{\mathcal{C}_{i}}(v, z)$ turns out to be independent of any complex structure modulus $z_{m}$. We want to note here that this does not mean that the complex structure moduli are flat directions for $W$ : there is still the denominator $D(z)^{2}$ in (1.2); furthermore it does also not mean that in such a case (of $\partial_{z_{m}} \operatorname{Pfaf} f_{\mathcal{C}_{i}}(v, z) \equiv 0$ everywhere $\forall i, \forall m$ ) the solution set in $\mathcal{M}=\mathcal{M}_{V} \times \mathcal{M}_{c x}$ has the structure of a certain region in $\mathcal{M}_{V}$ times the full $\mathcal{M}_{c x}$ factor. ${ }^{5}$ But we will be anyway generally not in such a situation.

Before we go on let us now remark that the special class of solutions we will investigate in this paper is indeed actually a class of solutions (cf. subset. 1.3 below): for if one has $\operatorname{Pfaf} f_{\mathcal{C}_{i}}(v, z)=0, \forall i$, for $(v, z)$ in some solutions set $S \subset \mathcal{M}=\mathcal{M}_{V} \times \mathcal{M}_{c x}$ and has that this vanishing happens even to second or higher order (such that also $\partial_{v_{l}} P f a f f_{\mathcal{C}_{i}}(v, z)=0, \forall i, \forall l$ and $\partial_{z_{m}} P f a f f_{\mathcal{C}_{i}}(v, z)=0, \forall i, \forall m$ hold in $S$ ) then indeed one will have $S \times \mathcal{M}_{K}$ (for the final factor cf. remark a) above) as a solution set for our main system of conditions $\partial_{v_{l}} W=\partial_{z_{m}} W=\partial_{k_{j}} W=W=0, \forall l, \forall m, \forall j$.

Connecting now the considerations of the last two paragraphs one finds that under the assumption that the Pfaffians are independent of the complex structure moduli one has ${ }^{6}$

\section{$\underline{\text { Solutions }}$}

with $\left\{\begin{array}{l}v \text { in a certain } \\ \text { region in } \mathcal{M}_{V}\end{array}\right\}$

times $\{$ flat cx. str. directions

\section{$\underline{\text { Solutions }}$}

where for $\left\{\begin{array}{l}(v, z) \text { in a certain } \\ \text { region in } \mathcal{M}_{V} \times \mathcal{M}_{c x}\end{array}\right\}$

one has $\left\{\begin{array}{c}\operatorname{Pfaf} f_{\mathcal{C}_{i}}=0 \text { for all } i \\ \text { to second or higher order }\end{array}\right\}$

However, the assumption $\partial_{z_{m}} P$ faf $f_{\mathcal{C}_{i}} \equiv 0$ is rather strong. One point of our specific explicit solution $\operatorname{set}^{7} \Sigma$ will be that it has flat $z_{j}$ directions (cf. also sect. 2.2) even without making such an extreme assumption: more precisely the solution set which we will find for each individual Pfaffian will have such a structure $\Sigma_{i} \times \mathcal{M}_{c x}$ (concerning notational accuracy here cf. later the footn. 19); this will be 'globalised', to make all Pfaffians vanish simultaneously, by going to the solution set $\Sigma \times \mathcal{M}_{c x}$ in $\mathcal{M}$.

\footnotetext{
${ }^{5}$ 'Solutions' include here always the vanishing at least to second order, i.e. we are speaking about the system $\partial_{k_{j}} W=\partial_{z_{m}} W=\partial_{v_{l}} W=W=0$.

${ }^{6}$ Here and in remark a) above when we speak of solutions with some flat directions - Kähler moduli or complex structure moduli - we mean of course only that the solution set contains a full factor $\mathcal{M}_{K}$ or $\mathcal{M}_{c x}$ (and not that $W$ generally would be independent of the corresponding moduli).

${ }^{7}$ which belongs to the wider, though still rather special class of solutions where one has vanishing to second or higher order - for each Pfaffian individually (for certain moduli); cf. also sect. 2.2
} 


\subsection{Résumé}

Above, in remark a) of sect. 2, we showed how, to be in a case where one has the freedom to have all possible Kähler moduli of $\mathcal{M}_{K}$ as part of the solution set of our original system (2.1) - (2.4) (2. order refers to the value and all first derivatives)

$$
W=\sum_{C} P_{C} e^{i k \cdot C}=0 \quad \text { to at least } 2 . \text { order }
$$

(like it happens for our specialised system (2.5) - (2.7)), characterises just those solutions for which already the individual $P_{C}$ vanish (to at least 2 . order). This is possible as the functions $e^{i k \cdot C}$ for the different homology classes are sufficiently 'separating' such that, having $W=0$ with the Kähler moduli $k_{j}$ in $k \cdot C=\sum k_{j} n_{j}^{(C)}$ nevertheless running completely unconstrained, can hold only if already the $P_{C}$ are zero individually (for some $(v, z) \in S_{\mathcal{M}} \subset \mathcal{M}_{V} \times \mathcal{M}_{c x} ;$ similarly for the derivatives).

One now would like to characterise in a second 'reduction step'8 our special solutions of $P$ faf $f_{\mathcal{C}_{i}}=0$ (to at least 2. order) among the already specialised class of solutions with

$$
P_{C}=\sum_{\left[\mathcal{C}_{i}\right]=C} \operatorname{Pfaf} f_{\mathcal{C}_{i}} D_{i}^{-2}=0 \quad \text { to at least } 2 \text {. order }
$$

The naive expectation would be that now the $D_{i}(z)$ play the role of the exponential factors and one is reduced to solutions where also the complex structure moduli run free.

However, two facts get in the way of this potential second reduction: the expressions $D_{i}(z)$ in the complex structure moduli are not given as explicitly as the exponentials in the Kähler moduli; secondly, the pfaffians $\operatorname{Pfaf} f_{\mathcal{C}_{i}}(v, z)$ itself depend also on the complex structure moduli $z_{m}$. This has the consequence that there are various possibilities which inhibit in general the intended reduction: there may be the case, for example, that the $\operatorname{Pfaf} f_{\mathcal{C}_{i}}$ (of a fixed class $C$ ) do not depend actually on the $z_{m}$ and that the $D_{i}$ are independent of the concrete instanton curve $\mathcal{C}_{i}$ of class $C$; then, easily, bundle moduli $v$ may exist for which $\sum_{i} \operatorname{Pfaf} f_{\mathcal{C}_{i}}(v)=0$ (to order $\geq 2$ ) which leads to $P_{C}=0$ (to order $\geq 2$ ) with the $z_{m}$ being able to run freely (unconstrained) without that one would need to have $\operatorname{Pfaf} f_{\mathcal{C}_{i}}(v)=0$ (to order $\geq 2$ ) individually. Another (theoretical) possibility is that the $z$-dependence in $\operatorname{Pfaf} f_{\mathcal{C}_{i}}(v, z)$ cancels that of $D_{i}(z)$ and one finds a $v$ as before.

So the set of solutions with $P_{C}=0$ (to at least 2. order) already (or equivalently solution set $\left.S=S_{\mathcal{M}} \times \mathcal{M}_{K}\right)$ has two distinct subsets, characterised by $S=S_{V} \times \mathcal{M}_{c x} \times \mathcal{M}_{K}$ and Pfaf $f_{\mathcal{C}_{i}}=0$, resp.. Our concrete solutions will lie in the intersection of these two sets.

\footnotetext{
${ }^{8}$ One highly exceptional case where some reduction can be effectuated is the case when all contributing instanton curves are distinguished in the sense that they are the only contributing curve in their class $C$ : then one finds indeed (from $D_{i}^{-2} \neq 0$ ) that our special solutions are just those fulfilling 'only' (2.10).
} 


\subsection{Dimension counting}

Before closing this propaedeutic section with its preliminary, formal remarks we want to state the dimension counting which shows how nontrivial in general is the idea to solve for supersymmetric Minkowski vacua (moduli leading to such vacua we call just 'solutions') by looking for critical points (including zero value) of all the individual Pfaffians of the instanton curves which contribute to the sum which builds the superpotential $W$.

Now, the ordinary approach or direct approach, involving just $W$ and its derivatives, leads to

$\underline{\text { General approach }} \quad h_{m}+1$ conditions $\quad$ for $\quad h_{m}$ moduli

where $h_{m}=h_{K}+h_{c x}+h_{V}$ counts the number of all moduli, comprising the Kähler, complex structure and bundle moduli. Here, as remarked earlier on, the additional condition comes from $W=0$, thus securing that the supersymmetric vacuum is Minkowski. So the system is only mildly overdetermined

By contrast, in our approach for the 'special solutions', involving all $N$ Pfaffians $\operatorname{Pfaf} f_{\mathcal{C}_{i}}$ and all of their first order derivatives, one has

'Special solutions' $N \cdot(h+1)$ conditions (with $N=\sharp\left\{\mathcal{C}_{i}\right\}$ ) for $h$ moduli (2.12) where $h=h_{c x}+h_{V}$ counts the number of moduli on which a Pfaffian depends, thus comprising only the complex structure and bundle moduli. Thus, as was to be expected, the system for the 'special solutions' shows a massive overdetermination. It seems to be a miracle how such a procedure should lead to solutions at all; and certainly the existence of such special solutions should, as remarked earlier on, only be possible if the conditions coming from the $N$ different instanton curves somehow 'fit together nicely'.

Now, what actually happens in our concrete examples for the occurrence of special solutions in certain set-ups, is the following. First a remark concerning our procedure. We treat in the following two main sections 3 and 4 actually two set-ups: $S U(n)$ spectral cover bundles on a Calabi-Yau space $X$ which is elliptically fibered over a base $B$ and then certain bundles defined in an analogy to the spectral construction for a general $X$. The first set-up will give us control only over instanton curves in $B$; but as the second construction in sect. 4 proceeds in a certain analogy to the spectral construction which is already well known we describe here the outcome for the first case, the spectral bundles on an elliptic $X$ of sect. 3 (so one has to keep in mind the caveat that the results in this case are only rudimentary as the concern actually only the part $W_{B}$ of $W$ build by contributions from base curves). 
Now, two simplifications (described here in the set-up of sect. 3) occur in our concrete example of such special solutions (despite our description both reductions occur at the same time). Consider first a single Pfaffian $P f a f f_{\mathcal{C}_{i}}(v, z)$. It turns out, unexpectedly, that there is no loss of dimensions connected with the demand that not only the value but also all first derivatives $\partial_{v_{l}} P f a f f_{\mathcal{C}_{i}}(v, z)$ and $\partial_{z_{m}} P f a f f_{\mathcal{C}_{i}}(v, z)$ vanish: the additional vanishing to second order can be tuned topologically, i.e. by choosing discrete parameters in a suitable manner ${ }^{9}$. So in this concrete case one gets a reduction of conditions (which concern the counting of degrees of freedom of the continuous parameters):

'Concrete special solutions' $N \cdot(h+1)$ conditions $\quad \longrightarrow \quad N \cdot 1 \quad$ conditions (2.13)

Also a second simplification occurs: the different conditions for all the individual Pfaffians related to the respective instanton curves $\mathcal{C}_{i}$ fit together nicely to a set of conditions which are independent of the respective $\mathcal{C}_{i}$ and can be posed for the global bundle $V$ once and for all. Thereby the number $N$, which is 'dangerous' as it can be quite large (examples show values in the thousands) and, more importantly, independent ${ }^{10}$ of the number of bundle (and complex structure) moduli, is reduced to a number which is related directly to bundle data (and independent of the number ${ }^{11} N$ ) and can be shown not to be greater than the relevant number $h$ of moduli (for the notation cf. sect. 3 ):

'Concrete special solutions' $N \cdot 1$ conditions $\longrightarrow(n-2) \frac{1}{2} \eta\left(\eta-n c_{1}\right)$ conditions $(2$

The mentioned final number of conditions (on continuous parameters) arises from the explicit description of the solution set which specialises (via $n-2$ divisibility conditions) the coefficients $a_{j}$ in the spectral cover equation of the cover surface $C$

$$
\mathcal{R}=\left\{t \in \mathcal{M}_{X}(C)\left|a_{n}\right| a_{j} \text { for } j=2, \ldots, n-1\right\}
$$

(this will be explained below). It gives the codimension in the continuous part of the bundle moduli space (for the concrete counting cf. remark a) after Theorem $P$ faf $f_{\text {all }} \subset B$ in sect. 3.4; for our concrete special solutions the complex structure moduli remain unconstrained; as for all special solutions the Kähler moduli remain also unconstrained).

As emphasized, all of this concerns the set-up of sect. 3 with its rudimentary results (pertaining only to base instanton curves); a corresponding discussion, proceeding along analogous lines, may now ensue for the proper case (pertaining to all instanton curves) of our original problem in the set-up of sect. 4 .

\footnotetext{
${ }^{9}$ cf. part b) of Theorem Pfaf $f_{\text {all }} \subset \subset$ B below in sect. 3.4

${ }^{10}$ in particular there is a priori no reason that $N$ should be not greater than $h$

${ }^{11}$ which depends mainly just on the geometry of $X$; only through the issue which of the $\mathcal{C}_{i}$ actually are contributing for $V$ (i.e. for which of them one has $P f a f f_{\mathcal{C}_{i}} \not \equiv 0$ ) it is related to $V$
} 


\section{A first try: spectral cover bundles on elliptic Calabi-Yau spaces}

To understand vanishing conditions for various Pfaffians it is helpful to discuss first in greater detail an explicit example. For this reason we start in this section by treating the case of a spectral cover bundle on an elliptically fibered Calabi-Yau space. Here we will be able to formulate quite definitive conditions for the vanishing of a single Pfaffian, in case it is related to an instanton curve in the base of the elliptic fibration. This will be then extended to a coherent treatment of the conditions for simultaneous vanishing of all the Pfaffians related to instanton curves in the base. The significance of this restricted success will be discussed below in sect. 3.6 and 3.7. We will go beyond this in sect. 4 .

So let us assume in this section that $X$ admits an elliptic fibration $\pi: X \rightarrow B$ which has a section ${ }^{12} \sigma$. Let us also make at first the (unreal) assumption that all potentially contributing isolated (smooth) rational curves $\mathcal{C}_{i}, i=1, \ldots, p$ lie in $B$; we will discuss the real, more complicated situation in the next section. Then $P$ faf $f_{\mathcal{C}_{i}}$ will depend on the moduli $v$ of $V$ only through the restriction $\left.V\right|_{\mathcal{E}_{i}}$ of $V$ to the elliptic surface $\mathcal{E}_{i}=\pi^{-1}\left(\mathcal{C}_{i}\right)$ over $\mathcal{C}_{i}$. Let us also assume that $V$ is an $S U(n)$ spectral cover bundle with spectral cover surface $C$ (of class $n \sigma+\pi^{*} \eta$ ), given by an equation (for standard technical details of the spectral cover description cf. app. A)

$$
w=a_{0}+a_{2} x+a_{3} y+\ldots+a_{n} x^{n / 2}=0
$$

(for $n$ even, say; the coefficients $a_{j}$ are sections over $B$ of suitable line bundles); so, apart from a discrete choice of a twist parameter $\lambda$, the moduli of $V$ are given by the motions of $C$ in $X$ (essentially, up to an overall scaling, they are the coefficients of the sections $a_{j}$; here by coefficients we mean the degrees of freedom, ordinary coefficients for $B=\mathbf{F}_{\mathbf{k}}$ )

$$
\mathcal{M}_{X}(C)=\mathbf{P} H^{0}(X, \mathcal{O}(C))
$$

The geometric meaning is that the bundle $V$ decomposes over the generic elliptic fibre $F$ as a direct sum of line bundles $\mathcal{O}_{F}\left(q_{i}-p\right)$ where the point $p$ is the zero point (where the section $\sigma$ meets $F$ ) and the $q_{i}, i=1, \ldots, n$ are some other points where $C$ meets $F$. These point positions on each fibre are the continuous moduli of the problem and are encoded by the (degrees of freedom of the) coefficients $a_{j}$ of the equation (3.1) for $C$.

The other discrete degree of freedom mentioned above, the twist parameter $\lambda$, arises as follows: when reconstructing the bundle $V$ from the fibrewise data it is still possible

\footnotetext{
${ }^{12}$ thereby $B$ will be considered to lie in $X$; we denote the co-/homology class of $B$ by $\sigma, F$ is the fibre; furthermore we denote $c_{1}(B)$ just by $c_{1}$ and usually will suppress pullbacks like in $\pi^{*} \eta$ for $\eta \in H^{1,1}(B)$
} 
to twist the construction by a line bundle $L$ on $C$ which is cohomologically fixed ${ }^{13}$ from our standing assumption $c_{1}(V)=0$ with the only remaining freedom in $c_{1}(L)$ being an element $\gamma \in \operatorname{ker} \pi_{C *}: H^{1,1}(C) \rightarrow H^{1,1}(B)$; but $\gamma$ can generically be only of the form

$$
\gamma=\lambda \Lambda=\left.\lambda \widetilde{\Lambda}\right|_{C}=\left.\lambda\left(n \sigma-\left(\eta-n c_{1}\right)\right)\right|_{C}
$$

One finds $\lambda$ to be a half-integral integer with precise integrality restrictions for the various cases ( $n$ even or odd). From here one can write $L$ explicitly as a line bundle $\left.\mathcal{O}_{X}(D)\right|_{C}$.

The moduli space $\mathcal{M}_{V}$ of connected component $\mathcal{M}_{X}(C)$ has dimension

$$
\begin{aligned}
\operatorname{dim} \mathcal{M}_{V} & =h^{0}\left(X, \mathcal{O}_{X}(C)\right)-1=\frac{1}{12}\left(c_{2}(C)+c_{1}^{2}(C)\right) C-1=\frac{1}{12}\left(c_{2}(X) C+2 C^{3}\right)-1 \\
& =n-1+\frac{n^{3}-n}{6} c_{1}^{2}+\frac{n}{2} \eta\left(\eta-n c_{1}\right)+\eta c_{1}
\end{aligned}
$$

Here we used the assumption that $C$ is ample (usually assumed so that $h^{0,1}(C)=0$ and $L$ has no continuous moduli). Below in (3.13) we write the dimension from the degrees of freedom provided by the coefficients $a_{j}$ in (3.1).

\subsection{Instanton curves $\mathcal{C}_{i}$ in the base $B$ and the relevant moduli}

Then the restrictions see only the motions of $c_{i}=C \cap \mathcal{E}_{i}$ in $\mathcal{E}_{i}$, given essentially by the restrictions $a_{j ; i}:=\left.a_{j}\right|_{\mathcal{C}_{i}}$ which are homogeneous polynomials on these $\mathbf{P}^{\mathbf{1}}$ 's. Now, from $\operatorname{Pfaf} f_{\mathcal{C}_{i}}(v, z)=0$ one gets ('narrow') conditions on all of the polynomial coefficients of the $a_{j ; i}$ which translate back to conditions on the $a_{j}$ (the 'more relaxed' conditions mentioned above as for each $\mathcal{C}_{i}$ only some subset of the moduli $a_{j}$ is seen by the restriction $\left.a_{j ; i}\right)$. Now the point is that it is not at all clear that posing all these conditions on $a_{j}$ for $i=1, \ldots, p$ simultaneously still allows for a solution. To allow for a common solution the conditions should better 'fit together nicely'. Clearly this can not be expected to happen in general; rather these conditions will usually turn out to be contradictory.

However, and this is the point of the present note, there are cases where the individual conditions from the $\mathcal{C}_{i}$ lead to a compatible set of conditions on the moduli of the global bundle $V$ because each of these conditions did express already not an 'accidental' zero for $\operatorname{Pfaf} f_{\mathcal{C}_{i}}$ but did represent a 'conceptual' zero: the latter fits into a global system of conditions in the sense that the individual conditions for the $\left.V\right|_{\mathcal{E}_{i}}$ were already parts (concretely restrictions) of one global condition on the bundle $V$ over $X$. This is the case we are going to consider.

\footnotetext{
${ }^{13}$ and even completely fixed up to the mentioned discrete choice if one assumes, as we do, that $H^{0,1}(C)=0$ (as would follow, for example, for $C$ being an ample divisor in $X$ from $H^{0,1}(X)=0$ )
} 


\subsection{The problem of skew curves}

However, there is a problem. In the spectral cover scenario one can get, as we are going to describe, some control over the Pfaffians for base curves $\mathcal{C}$ (as always smooth isolated rational), i.e. curves in the image of $i: B \rightarrow X$ (embedding via section). There are, of course, many other curves in $X$ relevant as instantons besides these 'horizontal' curves. Here we use the obvious terminology calling a curve of homology class $i_{*} \psi+k F$ 'horizontal' (or a base curve) for $k=0$ (these are just the curves in $B$ ), 'vertical' for $\psi=0$ (these [reducible for $k>1$ ] fibers are here elliptic and therefore not interesting for us), and finally we call them lying 'skew' in the remaining cases. There can, and will in general, exist smooth isolated rational curves in $X$ which are not lying in the base but are skew. The behaviour of the bundle, with respect to the triviality criterion concerning the Pfaffian, is out of control for such non-base curves (as one lacks the crucial relation $\left.V\right|_{\mathcal{C}_{i}}=\left.\pi_{*} L\right|_{c_{i}}$ from the spectral approach which is tied to the fibration structure over $\mathcal{C}_{i}$ ).

\subsection{A way out through a moduli split ?}

If one would know, however, that those moduli $v_{s}$ of $V$ which control the Pfaffians for the skew curves are distinct from the corresponding moduli $v_{b}$ for the base curves one could still argue that the common solution set ${ }^{14}$, where the Pfaffians vanish (to second order at least) in the 'base-relevant' moduli, constitutes a partial moduli fixing: for if one would have a decomposition $W=W\left(v_{b}\right)+W\left(v_{s}\right)$ this would mean that, when taking derivatives with respect to the $v_{b}$, the contribution from the $v_{s}$ does not matter. In [8] it was claimed indeed that, because generically the skew curves avoid to hit the base curve $e^{15}$, the corresponding moduli sets, relevant for the Pfaffian contributions, are disjoint.

As a minor caveat let us first remark that, of course, one would in principle (when stabilising moduli and just demanding that $W=0$ in total for the stabilised values) not have needed to ask for $W=0$ individually for the $W\left(v_{b}\right)$ part (as one does, however, here in this approach implicitly when using a second or higher vanishing order of the $W\left(v_{b}\right)$ part at its stabilised moduli). Doing so, one has then also to ask for $W\left(v_{s}\right)=0$ individually for the $W\left(v_{s}\right)$ part when stabilising the $v_{s}$ moduli (if one searches for moduli stabilised with $W=0$ for the total $W$ ). It might occur a case, however, that a solution exists which makes all the individual derivatives vanish but makes also only the total sum $W=W\left(v_{b}\right)+W\left(v_{s}\right)$ vanish and not the individual parts.

\footnotetext{
${ }^{14}$ this in our examples turns out always to be a whole submanifold and not just points

${ }^{15}$ there a single base curve is considered but the argument extends to the case of finitely many $\mathcal{C}_{i} \subset B$
} 
More fundamentally, however, it is not at all clear whether one should even expect that the set of all bundle moduli decomposes into sectors of moduli, relevant for the respective individual instanton curves, as soon as the latter are disjoint (a connection is made in [8] to the problem of fivebrane-transitions and their relevant moduli where a fivebrane curve (the compact part of the fivebrane) is understood as a limiting 'small instanton' object for the bundle and then dissolved into the bundle, enlarging its second Chern class correspondingly; but this is not investigated at all for skew curves).

Actually, the argument in [8] served, however, a different purpose which can be achieved in another way. There the emphasis was to show that, after finding nontrivial results for individual Pfaffians, the full $W$ is not nevertheless identically zero from a cancellation after summation over all curves. Concretely there was a single base instanton curve and one had to care that its nonzero contribution is not cancelled. For this one does not need, however, the mentioned unreal moduli split (cf. the discussion before Question $\mathrm{W}$ (universal) ${ }^{\prime}$ in the introduction). It is enough to note that a hypothetical fact $W \equiv 0$ from cancellation between nonzero summands nevertheless must mean that the individual $P_{C}=\sum_{[\mathcal{C}]=C} \frac{P f a f f_{\mathcal{C}}(v, z)}{D_{\mathcal{C}}(z)^{2}}$ already vanish identically (cf. the discussion earlier on). But obviously a base curve is homologically distinguished (by definition) from a skew curve.

\subsection{The moduli space and the solution set}

We now give the details concerning the actual solution sets in our set-up. We have a spectral cover bundle with (3.1) as equation of the cover surface $C$; we denote by $a_{n ; i}=\left.a_{n}\right|_{\mathcal{C}_{i}}=\prod_{k=1}^{r_{i}-n} a_{n ; i}^{(k)}$ the decomposition into linear factors of the highest coefficient $\left(r_{i}:=\eta \cdot \mathcal{C}_{i}\right)$. Further we define, following [11], in the moduli space $\mathcal{M}_{\mathcal{E}_{i}}\left(c_{i}\right)$ two subloci: first the locus $\mathcal{R}_{i}$ where all resultants $R_{j ; i}^{(k)}=\operatorname{Res}\left(a_{j ; i}, a_{n ; i}^{(k)}\right)$ for $j=2, \ldots, n-1$ and $k=1, \ldots, r_{i}-n$ vanish; this locus of codimension $(n-2)\left(r_{i}-n\right)$ is the locus where $a_{n ; i}$ is a factor of all the $a_{j ; i}$ for $j=2, \ldots, n-1$

$$
\mathcal{R}_{i}=\left\{t \in \mathcal{M}_{\mathcal{E}_{i}}\left(c_{i}\right)\left|a_{n ; i}\right| a_{j ; i} \text { for } j=2, \ldots, n-1\right\}
$$

Secondly the locus where $\mathcal{O}_{c_{i}}\left(\left.\Lambda\right|_{c_{i}}\right)$ becomes trivial (using the notation $\Lambda$ also as divisor)

$$
\Sigma_{i}=\left\{t \in \mathcal{M}_{\mathcal{E}_{i}}\left(c_{i}\right) \mid \mathcal{O}_{c_{i}}\left(\left.\Lambda\right|_{c_{i}}\right) \cong \mathcal{O}_{c_{i}}\right\}
$$

This means that as the moduli change (concretely as one has different coefficient sections $a_{j}$ in the defining equation (3.1) of $C$ ) one has different concrete divisors given by the surface $C$ in $X$, and correspondingly also different $c_{i}$; and as such a $c_{i}$ varies it may 
happen that the universal twist bundle ${ }^{16} \mathcal{O}_{C}(\Lambda)$ on $C$ becomes trivial on $c_{i}$ for certain moduli. As we recall in the next theorem this can be controlled on the one hand by an explicit condition (related to the locus $\mathcal{R}_{i}$ ) in the $a_{j}$ and on the other hand leads to an effectivity assertion for the divisor of the line bundle on $c_{i}$ which gives $\left.V\right|_{\mathcal{C}_{i}}$ when projected down to $\mathcal{C}_{i}$; this effectivity makes then the corresponding Pfaffian vanish as will be recalled in Theorem $P f a f f_{\text {single } C \subset B}$ below.

In a similar vein one defines corresponding notions pertaining to the whole of the global surface $B$ (not just to a sublocus given by an instanton curve $\mathcal{C}_{i}$ )

$$
\begin{aligned}
\mathcal{R} & :=\left\{t \in \mathcal{M}_{X}(C)\left|a_{n}\right| a_{j} \text { for } j=2, \ldots, n-1\right\} \\
\Sigma & :=\left\{t \in \mathcal{M}_{X}(C) \mid \mathcal{O}_{C}(\Lambda) \cong \mathcal{O}_{C}\right\}
\end{aligned}
$$

So in both cases - the situation over a single instanton curve $\mathcal{C}_{i}$ in $B$ and the situation over $B$ as whole (with all instanton curves $\mathcal{C}_{i} \subset B$ considered simultaneously) - we have defined two subloci of the relevant moduli space: the locus $\Sigma_{i}$ or $\Sigma$ has a conceptual description and is directly relevant to the vanishing of the Pfaffian; the set $\mathcal{R}_{i}$ or $\mathcal{R}$ has a completely explicit description. $\mathcal{R}_{i} \subset \Sigma_{i}$ or $\mathcal{R} \subset \Sigma$ is easily seen and it would suffice to state our theorems for $\mathcal{R}_{i}$ or $\mathcal{R}$; but it is useful to have the following (proven in app. A.3) Proposition One has the following equalities of loci in the respective moduli spaces

a) $\mathcal{R}_{i}=\Sigma_{i}$ in $\mathcal{M}_{\mathcal{E}_{i}}\left(c_{i}\right)$

b) $\mathcal{R}=\Sigma$ in $\mathcal{M}_{X}(C)$

After these preliminary remarks on the geometric significance of the various subloci in moduli space which we have defined let us state the relevance of these loci to our main problem, that is to the question of the vanishing loci (vanishing divisors), denoted $\left(P f a f f_{\mathcal{C}_{i}}\right)$, of the various Pfaffians over instanton curves. In this section we will have to restrict us to instanton curves $\mathcal{C}_{i}$ which lie in the base $B$. As above we will first give the result for an individual instanton curve $\mathcal{C}_{i}$, where we describe a subset of $\left(\operatorname{Pfaf} f_{\mathcal{C}_{i}}\right)$, and afterwards the globalised version describing the corresponding subset of $\bigcap_{\mathcal{C}_{i} \subset B}\left(P f a f f_{\mathcal{C}_{i}}\right)$.

In an investigation [11] about the conceptual meaning of some explicit components (and more general subloci) of the vanishing locus given by the zero divisor $\left(\operatorname{Pfaf} f_{\mathcal{C}_{i}}\right)$ of Pfaffians $\operatorname{Pfaf} f_{\mathcal{C}_{i}}$ in some concrete examples [7], the following Theorem was shown [11] (for convenience of the reader we recall the proof of part a) and c) of the theorem in app. B; note that the $S U(2)$ assertion of b) follows from a) as then $\mathcal{R}_{i}=\mathcal{M}_{\mathcal{E}_{i}}\left(c_{i}\right)$ as only $a_{0 ; i}$ and $a_{2 ; i}$ exist; for further interpretations and some caveats cf. sect. 3.6 and 3.7):

\footnotetext{
${ }^{16}$ A remark concerning notation: here $\Lambda$ denotes the divisor whose cohomology class was used in (3.3); this and corresponding occasional usage of common notation for some divisors and their cohomology classes will not cause any confusion
} 
Theorem $P$ faff $f_{\text {single } \mathcal{C} \subset B}$

a) The set $\Sigma_{i}$ is contained in the locus where $\operatorname{Pfaf} f_{\mathcal{C}_{i}}$ does vanish

$$
\Sigma_{i} \subset\left(\operatorname{Pfaf} f_{\mathcal{C}_{i}}\right)
$$

b) one has $\operatorname{Pfaff} \equiv 0$ for $n=2$

c) for the multiplicity $k$ of such a zero of $\operatorname{Pfaf} f_{\mathcal{C}_{i}}$, i.e. for the vanishing order, one has the lower bound (where $r_{i}=\eta \cdot \mathcal{C}_{i}$ )

$$
k \geq \begin{cases}1+\frac{n}{4}\left(r_{i}-\frac{n}{2}\right) & \text { for } n \equiv 0(2) \\ 1+r_{i}-\frac{n+1}{2} & \text { for } n \neq 0(2)\end{cases}
$$

Remark: To give an example for an estimate of the $r_{i}$ we choose the base $B$ to be a del Pezzo surface $\mathbf{d P}_{\mathbf{k}}$ (cf. appendix A.1): here one has from the standing assumption ${ }^{17}$ (also adopted here by us), that $C$ is not only an effective divisor but even ample, that $\eta-n c_{1}$ is even ample and so $\eta \cdot E_{i}>n$ for the exceptional blow-up curves $E_{i}$. But there is, of course, a total of 240 instanton curves on $\mathbf{d P}_{\mathbf{8}}$, say, not just the $8 E_{i}$.

Actually, however, one finds the derived bound for $r_{i}$ for all instanton curves in $B=$ $\mathbf{d P}_{\mathbf{k}}$ and even more generally for all instanton curves in all bases $B$ : this follows from $K_{B} \cdot \mathcal{C}_{i}=-2-\mathcal{C}_{i}^{2}$ and the fact that all instanton (i.e. smooth isolated rational) curves $\mathcal{C}_{i}$ have selfintersection $\mathcal{C}_{i}^{2}=-1$ so that one has from $\eta-n c_{1}$ ample that even in general

$$
r_{i}>n
$$

The conceptual identification given in (3.9) of a vanishing locus for the respective Pfaffians $P$ faf $f_{\mathcal{C}_{i}}$ for all the individual base instanton curves $\mathcal{C}_{i}$ now makes it possible to 'globalize' (over the base $B$ ) the result immediately: all individual Pfaffians can be made vanish simultaneously if one chooses the moduli ${ }^{18}$ (globally over $B$ and not just over an individual $\mathcal{C}_{i}$ ) to lie in the set $\mathcal{R}=\Sigma\left(\right.$ where $\mathcal{R} \subset \cap_{i} \mathcal{R}_{i}$ obviously $^{19}$ )

Theorem $P f a f f_{\text {all } c \subset B}$

a) For an $S U(n)$ spectral bundle $V$ on an elliptic Calabi-Yau threefold $X$ one finds common zeroes for the Pfaffians of all base curves $\mathcal{C}_{i}$ by choosing moduli from the set $\Sigma$

$$
\Sigma \subset \bigcap_{\mathcal{C}_{i} \subset B}\left(P f a f f_{\mathcal{C}_{i}}\right)
$$

b) one gets a suitable multiplicity $k \geq 2$ from (3.10) applied to $r:=\min _{i} r_{i}$ when one tunes the $r_{i}$ from a suitable choice of $\eta$ : this needs $r \geq 3$ for the $S U(4)$ case and $r \geq 3$ or 4 for the $S U(3)$ or $S U(5)$ case, respectively; from (3.11) this is fulfilled automatically.

\footnotetext{
${ }^{17}$ to have $C$ irreducible (and then $V$ stable) one assumes in any case $j u s t$ that $\eta-n c_{1}$ is effective

${ }^{18}$ the coefficients of the $a_{j}$ in a polynomial language for $B$ a Hirzebruch surface $\mathbf{F}_{\mathbf{m}}$, for example

${ }^{19}$ here obvious pullbacks res $_{i}^{-1} \mathcal{R}_{i}$ are understood (with the restrictions res $_{i}: \mathcal{M}_{X}(C) \rightarrow \mathcal{M}_{\mathcal{E}_{i}}\left(c_{i}\right)$ )
} 
Remarks: a) Along the solution locus $\mathcal{R}$ the degrees of freedom in $\mathcal{M}_{V}$ given by the sections ${ }^{20} a_{j}$ of $\mathcal{N} \otimes \mathcal{L}^{-j}$ over $B$ are, for $j=2, \ldots, n-1$, reduced to the freedom provided by sections $a_{j}^{\prime}$ of $\mathcal{L}^{n-j}$ (they are of class $(n-j) c_{1}$ ) in $a_{j}=a_{j}^{\prime} \cdot a_{n}$. We compare the dimensions ${ }^{21}$ of the full moduli space $\mathcal{M}_{V}$ (reproducing (3.4)) and of our sublocus $\mathcal{R}$ (producing (3.4) with the coefficient $\frac{n}{2}$ of $\eta\left(\eta-n c_{1}\right)$ replaced by 1 ): its codimension $(n-2) \frac{1}{2} \eta\left(\eta-n c_{1}\right)$ shows how much the partial moduli fixing has reduced the moduli freedom (cf. the remark concerning the situation for one instanton curve in app. A.2)

$$
\begin{aligned}
\operatorname{dim} \mathcal{M}_{V} & =h^{0}(B, \mathcal{N})+\sum_{j=2}^{n-1} h^{0}\left(B, \mathcal{N} \otimes \mathcal{L}^{-j}\right)+h^{0}\left(B, \mathcal{N} \otimes \mathcal{L}^{-n}\right)-1 \\
\operatorname{dim} \mathcal{R} & =h^{0}(B, \mathcal{N})+\sum_{j=2}^{n-1} h^{0}\left(B, \mathcal{L}^{n-j}\right)+h^{0}\left(B, \mathcal{N} \otimes \mathcal{L}^{-n}\right)-1
\end{aligned}
$$

b) For $t \in \mathcal{R}$ the $z \in \mathcal{M}_{c x}$ remain flat directions (as do the $k \in \mathcal{M}_{K}$, cf. remark in sect. 1.2.2): the conditions for $\mathcal{R}$ are posed in the $\mathcal{M}_{V}$ factor of $\mathcal{M}=\mathcal{M}_{V} \times \mathcal{M}_{c x}$ alone.

\subsection{Topological vanishing of the Pfaffian}

Sometimes one gets a vanishing result for a Pfaffian which is completely independent of the continuous moduli ('topological vanishing'). In that case the Pfaffian vanishes along a whole component of the moduli space. These components are labeled by the discrete degrees of freedom. One such result is of course the identical vanishing of the Pfaffian (for a base curve) for $S U(2)$ bundles mentioned in Theorem $P f a f f_{\text {single } C \subset B}$ above.

We have focused above exclusively on the continuous moduli, so let us treat here now also the discrete parameter $\lambda$ in (3.3) (and, to a certain extent, the discrete degrees of freedom encoded in the integral cohomology class $\eta$ ). First and foremost one realises that the Theorem $\operatorname{Pfaf} f_{\text {single } \mathcal{C} \subset B}$ is completely independent of $\lambda$. The reason, of course, is that the degree of freedom incorporated in $\lambda$ just multiplies the object whose triviality, along a certain locus in moduli space, is concerned (the possible half-integrality of $\lambda$ turns out to play no role effectively). So, here one gets a vanishing result for the Pfaffian which depends on specific continuous moduli but is independent of the discrete modulus $\lambda$ (the conditions on $r_{i}=\eta \cdot \mathcal{C}_{i}$ were given above).

But there are also 'opposite' cases (so to speak) where one gets a vanishing result for the Pfaffian which depends on specific choices of the discrete modulus $\lambda$ but is independent of the continuous moduli. This happens if the effectivity of the divisor $\left.\left(\alpha s_{i}+\beta_{i} F\right)\right|_{c_{i}}$,

\footnotetext{
${ }^{20}$ they have class $\eta-j c_{1}$; here we use the line bundle $\mathcal{L}:=K_{B}^{-1}$ of class $c_{1}$

${ }^{21}$ assuming here that besides $\eta-n c_{1}$ also $c_{1}$ is ample, i.e. $B=\mathbf{d P}_{\mathbf{k}}$, to use index computation
} 
related to the line bundle $\left.{ }^{22} L\right|_{c_{i}} \otimes \mathcal{O}_{c_{i}}\left(-\left.F\right|_{c_{i}}\right)=\left.\mathcal{O}\left(\alpha s_{i}+\beta_{i} F\right)\right|_{c_{i}}$, follows already from a corresponding effectivity of the divisor on $\mathcal{E}_{i}$, not yet restricted to $c_{i}$. Clearly in such a case one gets a vanishing result for $\operatorname{Pfaf} f_{\mathcal{C}_{i}}$ which is independent of the continuous moduli and depends only on the discrete choices.

To find these cases note first that one gets (from the first lines in (B.1)) from $\alpha \geq 0$ that $\lambda \geq-1 / 2$, more precisely ${ }^{23}$ that $\lambda \geq 0$ for $n$ even and $\lambda \geq-1 / 2$ for $n$ odd. One then implements in addition the condition $\beta_{i} \geq 0$. This gives, as cases independent of $r_{i}$, for $n$ even the case $\lambda=0$ and for $n$ odd the cases $\lambda=-1 / 2$ and $+1 / 2$ (note that these case were, for technical reasons, outside the considerations of $[7]$ and $[10,11])$.

Further inspection shows that there are in addition some exceptional cases. Let us first recall that we have the restriction $r_{i}>n$ (from our assumption $C$ ample, cf. app. A.1). The condition $\beta_{i} \geq 0$ gives the other bound $r_{i} \leq \frac{2 \lambda n-1}{2 \lambda-1}$ (we assume that we are now in the $\lambda \geq 1$ regime). The universal case of $S U(2)$ was mentioned above. The other cases of interest $n=3,4,5$, related to an $E_{6}, S O(10), S U(5)$ GUT group (in the visible sector), give then a list of exceptional cases

Theorem 2 Pfaff $f_{\text {all } \mathcal{C} \subset B}$

For an $S U(n)$ spectral bundle on an elliptic Calabi-Yau threefold the Pfaffians $\operatorname{Pfaf} f_{\mathcal{C}_{i}}$ of all base instanton curves $\mathcal{C}_{i} \subset B$ vanish identically in the following cases:

a) $\underline{n \text { odd : }} \quad \lambda= \pm 1 / 2$

$$
\underline{n \text { even : }} \lambda=0
$$

b) Furthermore there are the following exceptional cases (the $r_{i}$-conditions are for all $i$ ): $\underline{S U(2):}$ universally

$\underline{S U(3):} \lambda=3 / 2$ and $r_{i}=4$

$\underline{S U(4):} \lambda=1$ and $r_{i}=5$ or 7

$\lambda=2$ and $r_{i}=5$

$\underline{S U(5):} \lambda=3 / 2$ and $r_{i}=6$ or 7

$\lambda=5 / 2$ and $r_{i}=6$

Remarks: a) Note that by (A.12) these cases are not so special in $\lambda$ but just in $r_{i}$.

b) Often (if $B$ has many instanton curves) it will be difficult to arrange that all the $r_{i}=\eta \cdot \mathcal{C}_{i}$ fulfil these conditions: for example on the del Pezzo surfaces $\mathbf{d P}_{\mathbf{k}}$ with $k \geq 5$ one has (cf. app. A.1) as instanton curves the exceptional blow-up curves $E_{i}, i=1, \ldots, k$ and also curves of classes $l-E_{i}-E_{j}$ and $2 l-\sum_{n=1}^{5} E_{j_{n}}$ which illustrates this problem.

\footnotetext{
${ }^{22}$ for which one wants to establish the existence of a nontrivial section (cf. the step $\Sigma_{i} \subset\left(P f a f f_{\mathcal{C}_{i}}\right)$ in the proof of theorem $\operatorname{Pfaff} f_{\text {single } C \subset B}$ in app. B)

${ }^{23}$ as $\lambda$ is integral (and $r$ odd) for $n$ even and $\lambda$ is strictly half-integral for $n$ odd
} 


\subsection{The relevance of vanishing results for base instanton curves}

In the last two subsections we found vanishing results for all individual base instanton curves, either for a certain subset among the continuous moduli (what, for abbreviation, we will call henceforth 'the special case'), or just specified by discrete parameters (what we will call 'the component case'). This does not give, of course, a satisfying answer to

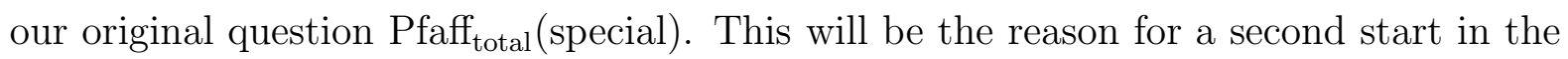
next section where we try to define bundles for which the corresponding argument can be made conclusive (i.e. to include all instanton curves, not just those in a base).

Nevertheless let us point out the impact of the given partial results. For this we have to isolate (as far as possible) in the general expression for the superpotential the directions belonging to the base and to the fibre, respectively. So, if $[\mathcal{C}]=\sum n_{j} C_{j}+n_{F} F$ is the decomposition of the homology class of an instanton curve in a homology basis (the $n_{j}$ and $n_{F}$ integral) and $J=\sum k_{m} J_{m}+k_{F} J_{F}$ a decomposition of a given Kähler class with respect to a basis duall ${ }^{24}$ to the $C_{j}$ (the $k_{m}$ and $k_{F}$ are the Kähler moduli), then one gets

$$
W=\sum_{l=0}^{\infty} a_{l} q^{l}=W_{B}+W_{\text {skew }}
$$

(with $q:=e^{i k_{F}}$ and $W_{B}=a_{0}$ ) where $a_{l}=\sum_{C} P_{C} e^{i \sum n_{j} k_{j}}$ and the summation over $C$ goes over classes which have fibre component part $n_{F} F=l F$.

Now, what the results up to now say, is just that the constant term (in $q$ ) of this series can be made to vanish: $a_{0}=0$. More precisely, this happens either for $t \in \mathcal{R}$ (with $\lambda$ arbitrary; this was our 'special case') or for one of the whole components (codimension zero) $\mathcal{M}_{X}(C)$ of the bundle moduli space specified by the discrete parameters as in Theorem 2 Pfaf $f_{\text {all } \subset \subset B}$ above (the 'component case'). What one learns from this is just that, at a certain boundary ${ }^{25}$ region of $\mathcal{M}_{K}$, more precisely for the partial decompactification limit of large fibre, one has $W=0$ (this holds with the bundle moduli specified as in the special case or the component case); note that one has for this region in any case $\partial_{k_{F}} W=0$. Moreover one has in this region, in both cases (special or component), that $\partial_{k_{j}} W=0$ (again the only nontrivial part of this assertion is the behaviour of the constant term where the assertion follows directly from our theorems above).

Thus the mentioned theorems (in both cases) provide indeed supersymmetric Minkowski vacua, but only in a 'generalised sense' because one is, in this partial decompactification limit, not in the bulk but in the boundary of the Kähler moduli space.

\footnotetext{
${ }^{24}$ so $\int_{\mathcal{C}_{j}} J_{m}=\delta_{j m}, \int_{F} J_{m}=0, \int_{C_{j}} J_{F}=0, \int_{F} J_{F}=1$

${ }^{25}$ so this will be in any case only a 'solution' in a generalised sense
} 


\subsection{The location of the solution set in the moduli space}

We now will have a closer look on the actual solution set, the subset $\mathcal{R}=\Sigma$ of (Pfaff), and its location in the moduli space $\mathcal{M}_{V}$. Recall that the latter has $\mathcal{M}_{X}(C) \cong \mathbf{P} H^{0}\left(X, \mathcal{O}_{X}(C)\right) \cong|C|$ (the linear system) as its continuous part and the discrete choices involved in $\eta$ and $\lambda$ as labels for the components.

Let us recall first that indeed the discrete choice for the half-integral number $\lambda$ labels distinct branches of the moduli space. This is in a sense obvious but becomes also particularly palpable from the following perspective: the other discrete choice, that of the class $\eta$, will lead in general to $\eta\left(\eta-n c_{1}\right) \neq 0$; in such a case it is clear from $\frac{1}{2} c_{3}(V)=$ $\lambda \eta\left(\eta-n c_{1}\right)$, cf. [12], that indeed bundles constructed from different $\lambda$ lie in different branches (components disconnected to each other) of the moduli space $\mathcal{M}_{V}$.

Now let us look what happens to the line bundle $L$ on $C$, from which $V$ is constructed as $p_{*}\left(p_{C}^{*} L \otimes \mathcal{P}\right)$, along the specific locus $\Sigma$ in the moduli space $\mathcal{M}_{X}(C) \cong \mathbf{P} H^{0}\left(X, \mathcal{O}_{X}(C)\right)$ where $\left.\mathcal{O}_{X}(\widetilde{\Lambda})\right|_{C}=\left.\mathcal{O}_{X}\left(n \sigma-\left(\eta-n c_{1}\right)\right)\right|_{C}$ becomes trivial. There one has from

$$
L=\left.\mathcal{O}_{X}\left(n\left(\lambda+\frac{1}{2}\right) \sigma+\left[\left(n \lambda+\frac{1}{2}\right) c_{1}-\left(\lambda-\frac{1}{2}\right) \eta\right]\right)\right|_{C}
$$

the following specialisations ${ }^{26}$

$$
L \stackrel{\Sigma}{\rightarrow} \begin{cases}\left.\mathcal{O}_{X}\left(\frac{n}{2} \sigma+\frac{\eta+c_{1}}{2}\right)\right|_{C} & \text { for } n \text { even } \\ \left.\mathcal{O}_{X}\left(\eta-\frac{n-1}{2} c_{1}\right)\right|_{C} & \text { for } n \text { odd }\end{cases}
$$

Therefore, regardless of their specific form, the dependence on the parameter $\lambda$ has dropped out completely! This would mean that (for fixed $n$ and $\eta$ ) all the 'distinct' components with continuous part $\mathcal{M}_{X}(C)$ and just different discrete labels $\lambda$ are connected via the subset $\Sigma$ in which they meet. This, clearly, can mean only that $\Sigma$ actually does not lie in the bulk of $\mathcal{M}_{X}(C)$ but rather in its boundary. Therefore the supersymmetric solutions to the system $D W_{B}=W_{B}=0$ (where $W=W_{B}+W_{\text {skew }}$ ) we did find are solutions only in a generalised sense as they lie on a 'degeneration boundary' of $\mathcal{M}_{X}(C)$ (itself the continuous part of $M_{V}$ ), which, however, is not an uncommon thing.

Let us try to understand this phenomenon more directly: from a degeneration of $C$, enforced when going to $\Sigma$. For this we concentrate on the explicit description $\mathcal{R}$ of $\Sigma$ which contains directly the defining coefficient sections $a_{j}$ of the spectral cover equation and is therefore most accessible for an investigation which wants to find out the singularity of the corresponding spectral surfaces.

\footnotetext{
${ }^{26}$ Recall that for $n$ even one has $\lambda$ integral and $\eta \equiv c_{1}(2)$ as the case of a strictly half-integral $\lambda$ is excluded (it would need an even $c_{1}$ which itself is excluded by $\chi_{i}=\mathcal{C}_{i} \cdot c_{1}=1$ ).
} 
It is enough to illustrate what happens for the three cases of greatest interest, i.e. the $S U(3), S U(4)$ and $S U(5)$ bundles. They have respective affine cover equations $w_{5}=$ $a_{0}+a_{2} x+a_{3} y+a_{4} x^{2}+a_{5} x y$, with $w_{4}$ (or $w_{3}$ ) arising by setting $a_{5}$ (or $a_{5}$ and $a_{4}$ ) to zero. So one has (the mentioned specialisations give the corresponding results for $w_{3}$ and $w_{4}$ )

$$
\begin{aligned}
& \partial_{x} w_{5}=a_{2}+2 a_{4}+a_{5} y \\
& \partial_{y} w_{5}=a_{3}+a_{5} x
\end{aligned}
$$

The surface $C$ is the intersection ${ }^{27}$ of the elliptic Weierstrass equation and the spectral cover equation $w=0$. So along the locus $\mathcal{R}$ in moduli space the expression $w$ (in the affine patch described) has vanishing gradient at $\pi_{C}^{-1}(A)$ where $A=C \cap \sigma$ is the vanishing divisor of $a_{n}$ in $B$ (cf. A.19; $\pi_{C}=\left.\pi\right|_{C}$ ), so $C$ is singular there. As explained in the remark in app. A.3 one has here $\pi_{C}^{-1}(A)=n A$, so $A$ is a total ramification locus for $\pi_{C}$.

remark: Two all-important caveats: 1) a comprehensive degeneration dictionary between $C$ and $V$ has not been worked out; 2) we assume that our reasonings remain valid despite the degeneration (often this is so by continuation from the interior of the moduli space).

So, remarkably, (for fixed $n$ and $\eta$ ) this singular degeneration of $C$, which lies at a certain boundary location of the continuous part $\mathcal{M}_{X}(C)$ of the multi-component space $\mathcal{M}_{V}$, connects all the different components labeled by the parameter $\lambda$ (as being common to all of them, cf. after (3.17); one convinces oneself also about the opposite inclusion $)^{28}$

$$
\Sigma_{n, \eta}=\bigcap_{\lambda} \overline{\mathcal{M}(V)_{n, \eta}^{\lambda}}
$$

In particular, at this locus in the common (!) boundary of all the $\lambda$-components the amount of chiral matter can be arbitrarily switched (in units of $\eta\left(\eta-n c_{1}\right)$ ). This chirality change phenomenon should not come as a surprise because the chiral matter is known [12] to be related to the curve $A$. That is, when going to this common boundary locus, all the different generation numbers labeled by $\lambda$ jump so that - via going through this singular configuration - all generation numbers are connected (which might have interesting consequences for phenomenology; at the same time this 'jumping locus' is singled out as a specific supersymmetric solution, though just for the $W_{B}$ part and only in the described generalised sense as it lies at a degeneration boundary).

\footnotetext{
27 inside the fourfold given by the $\mathbf{P}^{\mathbf{2}}$ bundle, with projective coordinates $(x, y, z)$, over $B$

${ }^{28}$ Many statements throughout the paper, like whether $t \in \mathcal{M}_{X}(C) \subset \mathcal{M}_{V}$ or actually $t \in \overline{\mathcal{M}_{X}(C)} \subset$ $\overline{\mathcal{M}_{V}}$, have to be read with this bulk versus boundary question in mind; only in the present subsection did we make this distinction notationally manifest. The question of when (i.e. for which modulus) and where (in $C$ ) a spectral object $C$ becomes singular has to be investigated case by case: the distinction (like $\Sigma \subset \overline{\mathcal{M}_{X}(C)}$, with $C$ singular at $A$ for $t \in \Sigma$ ) could then be made manifest also in the corresponding statement. Also all statements have to be read with the assumption of the caveat remark above in mind.
} 


\section{A new class of bundles}

As we have seen, our approach - to search for critical points of the world-sheet instanton superpotential $W$ by searching for critical points of the individual Pfaffians for all contributing instanton curves - did have, when applied to spectral cover bundles on elliptically fibered $X$, only partial success: only the base instanton curves $\mathcal{C}_{i} \subset B$ were under control. Therefore we now make a fresh start and define a new class of bundles, now on an arbitrary Calabi-Yau threefold (not necessarily elliptically fibered), where we want to extend the previous method to a set-up where all instanton curves are under control.

\subsection{The idea of the construction}

The idea of the construction to follow is, of course, to use the control which one has over the Pfaffians for base instanton curves, in the spectral cover approach for spectral bundles over elliptic Calabi-Yau spaces $X$, now for all instanton curves in an arbitrary, not necessarily elliptically fibered $X$. For this let us first note that in sect. 3 the whole elliptic fibre direction played only a rather marginal role: we considered only base instanton curves $\mathcal{C}_{i} \subset B$ and so were only interested in the behaviour of the bundle when restricted to $B$ where the relation (or its versions restricted to the situation over a curve $\mathcal{C}_{i}$ )

$$
\left.V\right|_{B} \cong \pi_{C *} L
$$

was crucial. The whole fibre direction, i.e. the whole manifold $X$ beyond just the base $B$, was only important as an ambient space where a cover manifold $C$ of $B$ lives. The line bundle $L$ on $C$ was essentially fixed by our condition $c_{1}(V)=0$. The ambient space $X$ was then important for an explicit description, via the equation (3.1), of the possible concrete incarnations of $C$ in its linear system. This allowed to describe the (continuous) moduli of $V$ directly as $\mathcal{M}_{X}(C) \cong \mathbf{P} H^{0}\left(X, \mathcal{O}_{X}(C)\right)$.

Therefore it should be clear which parts of the spectral cover construction are actually responsible for its (partial) success: when controlling the Pfaffians for base instanton curves $\mathcal{C}_{i} \subset B$ one does not need to have any information about the behaviour of the bundle $V$ on $X$ outside of $B$. The fibrewise description of $V$ starting from the Poincare line bundle is not needed. The only impact of $C$ is described in (4.1).

So, if we want to have control over all instanton curves in an arbitrary (not elliptic) $X$, we just need to consider the bundle $V$ on $X$ as such a base part of a set-up in a fictitious elliptic fourfold $Y$ (not Calabi-Yau) over $X$ where we just demand that our bundle arises as a projection (a push forward) from a line bundle on a (threefold) cover $C$ as in (4.1). 
The ambient space $Y$ serves only as an auxiliary space to have a class of possible covers $C$ under control; i.e., the motions of $C$ in $Y$ will map to the moduli of $V$ on $X$.

\subsection{New classes of bundles}

Let us first explain our emphasis in the notion of what is 'new' with defining the classes of bundles below. The possibility to define such bundles is not new. The point is that we single out these bundles as objects to be studied because it turns out to be possible to apply the ideas of the previous section to gain control now over all Pfaffians; this gives these classes certainly a special importance.

One may give the relevant definitions first quite generally in the category of smooth compact complex Kahler manifolds (one may also think already of the algebraic category) of arbitrary dimension $k$ and holomorphic bundles.

\section{Definition 'Projected Bundles'}

A $U(n)$ vector bundle $V$ on an $k$-fold $X$ is called 'projected' if there is a (ramified) covering $k$-fold $p_{C}: C_{k} \rightarrow X$ and a line bundle $L$ on it such that $V \cong p_{C *} L$.

One would also like to know whether a given bundle is of this type; this leads to the question of giving an internal characterisation of a bundle $V$ over $X$ to be projected.

If $n$ is the covering degree of $p_{C}: C \rightarrow X$, such that $r k(V)=n$, one finds ${ }^{29}$ for a projected bundle $S U(n)$ bundle (i.e. for simplicity we assume $\left.c_{1}(V)=p_{*}\left(l+\frac{1}{2} c_{1}(C)\right) \stackrel{!}{=} 0\right)$

$$
\begin{aligned}
& c_{2}(V)=-p_{*}\left(\frac{1}{2} l^{2}+\frac{1}{2} l c_{1}(C)+\frac{c_{2}(C)+c_{1}^{2}(C)}{12}\right) \\
& c_{3}(V)=p_{*}\left(\frac{1}{3} l^{3}+\frac{1}{2} l^{2} c_{1}(C)+l \frac{c_{2}(C)+c_{1}^{2}(C)}{6}+\frac{c_{1}(C) c_{2}(C)}{12}\right)
\end{aligned}
$$

(where $l:=c_{1}(L)$ and $p:=p_{C}$ ). Usually one will use these results in a slightly different form where one makes manifest the condition $c_{1}(V)=0$ by the following choice of $l$

$$
l=-\frac{1}{2} c_{1}(C)+\gamma_{3}
$$

Here $\gamma_{3}$ is assumed to be in the kernel of $p_{*}: H^{1,1}(C) \rightarrow H^{1,1}(X)$.

In the situation where the topological type of $C$ is fixed (for more discussion about the possible variation of the cover threefold $C$ cf. below) all the dependence of the Chern classes on the input parameters of the construction lies therefore in this class $\gamma_{3}$. One

\footnotetext{
${ }^{29}$ with the Grothendieck-Riemann-Roch formula $\operatorname{ch}(V) \operatorname{Td}(X)=p_{*}(\operatorname{ch}(L) \operatorname{Td}(C))$
} 
finds then (where we use the abbreviations $c_{i}:=c_{i}(C), \gamma:=\gamma_{3}$ )

$$
\begin{aligned}
& c_{2}(V)=-\frac{1}{2} p_{*}\left(\gamma^{2}+\frac{2 c_{2}-c_{1}^{2}}{12}\right) \\
& c_{3}(V)=p_{*}\left(\frac{1}{3} \gamma^{3}+\frac{2 c_{2}-c_{1}^{2}}{12} \gamma\right)
\end{aligned}
$$

Further specification of the classes $c_{i}$ and $\gamma$ will arise in the constructions defined below.

Refined constructions

To have better control over the possible $C_{k}$, that is to describe their possible moduli more explicitly, we will also consider some refinements. For this note first that one may consider our definition in loose analogy to another class of bundles, introduced also in connection with the question to gain control over the contribution from the Pfaffians of all the world-sheet instantons [3]. There one has also a second space $Z$, endowed with a bundle $V_{Z}$, and a map $i: X \rightarrow Z$. From these data the given bundle $V$ on $X$ is derived

$$
\begin{aligned}
i: X & \rightarrow Z \\
V & \cong i^{*} V_{Z}
\end{aligned}
$$

Here $i$ is an embedding of $X$ as a hypersurface (or complete intersection) in $Z$ and $r k V=r k V_{Z}$. In our (in a sense 'dual') case one has

$$
\begin{aligned}
p: C & \rightarrow X \\
V & \cong p_{*} L
\end{aligned}
$$

Here $p$ is a projection which is a finite (ramified) covering and $r k L=1$.

To make actual progress with the investigation of a projected bundle $V$ one has to assume more structure on the auxiliary space, i.e. $Z$ in [3] and $C$ here: just as $Z$ is assumed to be a toric variety, here $C$ will be assumed to be describable as follows

\section{Definition 'Embedded Projected Bundles'}

A projected bundle $V$ on $X$ is called 'embedded projected' if there exists an embedding $i_{C}: C_{k} \rightarrow Y_{k+1}$ of $C_{k}$ in an $k+1$-fold $Y$

(Again one may try to characterise this class of bundles more intrinsically.) Now, often one will be in a situation where the line bundle $L$ on $C$ is fixed (up to discrete degrees of freedom) already by its cohomology class (the first Chern class) and has no further continuous moduli. In that case the moduli come from the possible motions of $C$ in $Y$ (i.e. the possible actual hypersurfaces in the given linear system). So one gets then a projection $\mathcal{M}_{Y}(C) \cong \mathbf{P} H^{0}\left(Y, \mathcal{O}_{Y}(C)\right) \rightarrow \mathcal{M}_{X}(V)$. 
To get further information on the degrees of freedom of the hypersurface $C$ in $Y$ one puts additional structure on $Y$, like the following (needless to say, again it would be of interest to characterise the class intrinsically)

\section{Definition 'Fibered Embedded Projected Bundles'}

A projected bundle $V$ on $X$ is called 'fibered embedded projected' if there exists an embedding $i_{C}: C_{k} \rightarrow Y_{k+1}$ of $C_{k}$ in an $k+1$-fold $Y$ which has a fibration $\pi: Y \rightarrow X$

We will usually add here the condition that the fibration has a section and $X$ is considered as divisor in $Y$. A we consider $C$ in the embedded case just as a hypersurface in $Y^{30}$ one has then, in the generic case (which we assume) where just $X$ and $\pi^{*} S$ are available, for the cohomology class $^{31}$ of the divisor $C$ in $Y$ (with $S \in H_{4}(X)$ )

$$
C=n X+\pi^{*} S
$$

\subsection{Elliptically embedded projected bundles}

In the class of fibered embedded projected bundles one may distinguish according to the genus of the fibre: a ruled case, an elliptic case and so on. We take as the class which is related most directly to the set-up we have in mind the following

\section{Definition 'Elliptically Embedded Projected Bundles'}

A projected bundle $V$ on $X$ is called 'elliptically embedded projected' if there exists an embedding $i_{C}: C_{k} \rightarrow Y_{k+1}$ of $C_{k}$ in an $k+1$-fold $Y$ which is elliptically fibered $\pi: Y \rightarrow X$ over $X$ with $X$ as a section.

Again one may ask for an internal understanding of this class, that is, in refinement of the earlier characterisation issue, one would now like to have an internal characterisation of a bundle $V$ over $X$ to be elliptically embedded projected.

Concretely $Y$ will be described by a Weierstrass equation ${ }^{32} z y^{2}=4 x^{3}-g_{2} x z^{2}-g_{3} z^{3}$ in a $\mathbf{P}^{2}$ bundle $\mathbf{P}\left(\mathcal{L}^{2} \oplus \mathcal{L}^{3} \oplus \mathcal{O}\right)$ over $X$ where $\mathcal{L}$ is a line bundle over $X$ (which we assume to be nontrivial; note that no bundle on $Y$ does occur here, no fibre product, no Poincare bundle). The hypersurface $C \subset Y$ will be described by an equation (for $n$ even, say)

$$
w=a_{0}+a_{2} x+a_{3} y+\ldots+a_{n} x^{n / 2}=0
$$

\footnotetext{
${ }^{30}$ in the following we suppress the dimension subscript and write just $C$ and similarly $p$ for $p_{C}=\left.\pi\right|_{C}$

${ }^{31}$ if no confusion can arise we denote the cohomology class of $C$ or $X$ in $Y$ just by $C$ and $X$, resp.; corresponding notations will be used below

${ }^{32}$ with $x, y, z, g_{2}, g_{3}$ sections of $\mathcal{L}^{k}$ for $k=2,3,0,4,6$; the $x, y, z$ being homogeneous coordinates of $\mathbf{P}^{2}$
} 
(using affine $x, y$ ) with the $a_{i}$ sections of $\mathcal{O}_{X}(S) \otimes \mathcal{L}^{-i}$. We will use the abbreviations

$$
\begin{aligned}
& c_{1}(L)=l \in H^{2}(C) \\
& c_{1}(\mathcal{L})=\lambda \in H^{2}(X)
\end{aligned}
$$

Clearly, the restrictions to the base $B_{2}$ or to a curve $\mathcal{C}$ in it of a spectral cover bundle in an elliptic Calabi-Yau threefold are examples of embedded projected bundles. What is new is that we will apply now this set-up to the bundle $V$ on our arbitrary Calabi-Yau threefold $X$ (which is our case from now on) as a whole.

For an elliptically embedded projected bundle one finds ${ }^{33}$ from the equation for $C$

$$
\begin{aligned}
& c_{1}(C)=-\left.(n X+S+\lambda)\right|_{C} \\
& c_{2}(C)=\left.\left(c_{2}(X)+12 \lambda X+11 \lambda^{2}+(n X+S)^{2}+\lambda(n X+S+\lambda)\right)\right|_{C}
\end{aligned}
$$

Taken together (4.2), (4.2) and (4.15), (4.16) give necessary conditions on $c_{k}(V)$ for an $S U(n)$ bundle $V$ to be elliptically embedded projected (as we assume from now on).

The degree of freedom introduced by the twisting line bundle $L$ on $C$ can be described more explicitly: one finds (cf. (4.4); suitable pullbacks to $Y$ for $S$ and $\lambda$ understood)

$$
l=\left.\frac{n X+S+\lambda}{2}\right|_{C}+\gamma_{3}
$$

where $\gamma_{3}$ is in the kernel of $p_{C *}: H^{1,1}(C) \rightarrow H^{1,1}(X)$. Generically - and we will assume that this generic class is the one actually used - this $\gamma_{3}$ will be just a multiple, $\mu$ say, of $n X-\left(S-n \lambda\right.$ ) (understood as class in $Y$ restricted to $C$ ); here $\mu \in \frac{1}{2} \mathbf{Z}$ with actually

$\mu \in \frac{1}{2}+\mathbf{Z}$ for $n$ odd, while for $n$ even $\mu \in \frac{1}{2}+\mathbf{Z}$ needs $\lambda$ even and $\mu \in \mathbf{Z}$ needs $S+\lambda$ even. From here one can write $L$ (even uniquely for $H^{0,1}(C)=0$, which itself would follow from $H^{0,1}(Y)=0$ and $C$ ample in $Y$ ) as a line bundle $\left.\mathcal{O}_{Y}(D)\right|_{C}$.

The genericity assumption made here concerning the concrete form of the twist class $\gamma_{3}$ will turn out to be so important that we want to state it explicitly

\section{Definition 'Generic Elliptically Embedded Projected Bundles'}

An eliptically embedded projected bundle $V$ is called generic if the twist class $\gamma_{3}$ of the line bundle $L$ on $C$ is built from the generic divisors only.

These generic divisors on $Y$ are $X$ (embedded as section) and the pullbacks $\pi^{*} S$ for $S$ a divisor in $X$. As the concrete form of the twist played a crucial role in the proof of Theorem Pfaf $f_{\text {single } \mathcal{C} \subset B}$, cf. app. B, on which our generalization will be modelled, we restrict us in the following to this generic class (in the described sense; cf. also sect. 4.3.2).

\footnotetext{
${ }^{33}$ note that $X^{2}=-\lambda X$; here the right hand sides in the expressions for $c_{k}(C)$ are - with some suppressed pullbacks from $X$ to $Y$ included - in $H^{*}(Y)$, and then restricted to $C$
} 
It will be important to determine the region in the Kahler cone where an embedded projective bundle is stable, in particular whether this region is nonempty ${ }^{34}$.

\subsubsection{An example}

In the special case that $X$ itself is actually elliptically fibered (with section) there is an important special class of bundles, the spectral bundles, which can be characterised abstractly as being fibrewise semistable (concretely: a sum of line bundles of degree zero). Such bundles have a non-empty stability region in the Kähler moduli space $\mathcal{M}_{K}$. They turn out to be of elliptically embedded projected type, but are not generic in our sense here as their twist line bundle involves the Poincare bundle which itself involves the diagonal class which is a non-generic divisor (in the sense used here; cf. sect. 4.3.2).

\section{Fact}

On an elliptically fibered Calabi-Yau threefold $X$ (with section) the spectral bundles are examples of elliptically embedded projected bundles $V$. They are stable but not generic.

So consider an elliptic $X$, with fibration $\pi_{B}: X \rightarrow B$ of fibre $F$ and section $\sigma$ and with a spectral bundle $V$ (with spectral surface $C_{2} \subset X$ of class $n \sigma+\pi_{B}^{*} \eta$, endowed with a line bundle $L_{2}$ ). For such a bundle we use ${ }^{35} C_{3}:=X \times_{B} C_{2}$ (of class $n X_{1}+\pi^{*}\left(\pi_{B}^{*} \eta\right)$ ), $Y:=X \times_{B} X$ and the line bundle on $C_{3}$ (with the Poincare bundle $\mathcal{P}$ on $Y$, cf. below)

$$
L_{3}:=p_{C_{2}}^{*} L_{2} \otimes \mathcal{P}
$$

Correctly interpreted, the equation for $C_{3} \subset Y$ is the same as the one for $C_{2} \subset X$ and $\mathcal{M}_{Y}\left(C_{3}\right) \cong \mathcal{M}_{X}\left(C_{2}\right)$. Choosing $C_{2}$ ample in $X$ makes $C_{3}$ ample in $Y$. Considering the first factor $X_{1}$ in $Y$ as the Calabi-Yau space $X$ we actually consider, the elliptic fibration from the second factor $X_{2}$ in $Y$ is the auxiliary fibration (over $X_{1}$ ) of the ambient space $Y$ of $C_{3}$. The cohomology class $\lambda$ of (4.14), related to this fibration $\pi=\pi_{1}$ of $Y$ over $X_{1}$, is the same as for the fibration of $X_{1}$ over $B$ : it is the class $\pi_{B}^{*} c_{1}$.

\subsubsection{On the relevance of the genericity assumption}

The definition of genericity given earlier may seem natural enough; nevertheless the proper importance of this concept in our investigation lies in the following reasoning. Our

\footnotetext{
${ }^{34}$ This will be sufficient in our set-up: we do not have to care that the stability region of $V$ in $\mathcal{M}_{K}$ includes specific Kahler moduli values which are potentially forced on us by moduli fixing from $W$; for in our procedure the vanishing of all the $P_{C}$ (even of all individual Pfaffians), at the locus in $\mathcal{M}$ we are going to describe, clearly leaves the Kahler moduli completely unfixed as remarked earlier on.

${ }^{35}$ actually $C_{3}$ has singular points which are discussed in [12] together with a resolution one can use
} 
strategy to gain control over all instanton curves on a general $X$ is to adopt the proof of theorem $\operatorname{Pfaf} f_{\text {single } C \subset B}$ (which gave control over the contribution of base instanton curves for a spectral bundle). When one goes through the analogy on which this procedure relies (cf. the only slight deviations in app. C compared to app. B; just a curve-dependent $\chi_{i}$ is implemented in addition) one finds that the argument for $\mathcal{R}_{i} \subset \Sigma_{i}$ is generally true but the second step $\Sigma_{i} \subset\left(P f a f f_{\mathcal{C}_{i}}\right)$ makes use of the concrete form of $\left.L\right|_{c_{i}}$. This in turn came from the representation (4.17), more precisely from the concrete form of the gamma class $\gamma=\left.\mu(n X-(S-n \lambda))\right|_{C}$ whose triviality (when restricted to $c_{i}$ ) is assured along $\Sigma_{i}$ in moduli space (meant is: the corresponding divisor class on $c_{i}$ becomes linearly equivalent to zero along $\Sigma_{i}$; this leaves the line bundle of an effective divisor, having a non-trivial section, thus concluding the argument). The form of $\gamma$ arose because the divisors used ( $X=X_{1}$ and $\pi^{-1} S$, for $S \subset X$ a divisor) are the only ones available in general.

However, the concrete form (4.18) above, related to the divisor $p_{C_{2}}^{*} c_{1}\left(L_{2}\right)+\Delta-X_{1}-$ $X_{2}+K_{B}$ shows that in $p_{C_{2}}^{*} c_{1}\left(L_{2}\right)$ all classes are of the desired form but in $c_{1}(\mathcal{P})=$ $\Delta-X_{1}-X_{2}+K_{B}$ (as divisor) only the classes $X_{1}, X_{2}=\pi^{-1} B$ and $K_{B}$ (which means of course $\pi^{-1} \pi_{B}^{-1} K_{B}$ ) are of this form; the diagonal $\Delta$ is not of this type. Comparing (4.17) and (4.18) one has $\gamma_{3}=p_{C_{2}}^{*} \gamma_{2}+c_{1}(\mathcal{P})$. For $p_{C_{2}}^{*} \gamma_{2}$ the usual argument applies: it is made linear equivalent to zero when $C_{3}$ is tuned in its moduli space appropriately (from tuning $C_{2}$ in its moduli space). The remaining terms, however, in particular the diagonal, show that our assumption about the form of $c_{1}(L)$, expressed after $(4.17)$, is violated.

Moreover it is not possible to extend the original argument to include this case as one can not tune $C_{3}$ such that $\left.c_{1}(\mathcal{P})\right|_{C_{3}}$ (as divisor) becomes linearly equivalent to zero: one has $c_{1}(\mathcal{P}) \cdot C_{3}=c_{1}(\mathcal{P}) \cdot \eta$, and intersecting with a suitable further class $\pi^{-1} \pi_{B}^{-1} \psi$ gives the class $\Delta-F_{1}-F_{2}$ (over all points of $\eta \psi$ in the respective fibre surface ${ }^{36}$ ); intersecting this further with $\Delta$ gives $0-1-1 \neq 0($ as $e(\Delta)=0$ for the elliptic diagonal).

\subsection{The moduli space and the solution set}

The mentioned twist-parameter $\mu$ gives a discrete degree of freedom in the construction. Given the way how $V$ is defined as embedded projected bundle its continuous moduli come from the deformations of $C$ in $Y$, i.e. from the choice of the concrete equation (4.12) for $C$. So, keeping $\mu$ fixed, one has a map ${ }^{37}$

$$
P: \mathcal{M}_{Y}(C) \cong \mathbf{P} H^{0}(Y, \mathcal{O}(C)) \rightarrow \mathcal{M}(V)
$$

\footnotetext{
${ }^{36}$ the product of the two elliptic fibers $F_{1}$ and $F_{2}$; we use the same symbol for various diagonal classes

${ }^{37}$ had we constructed a spectral cover bundle $V_{Y}$ over $Y$ from $C_{3}$ with Poincare bundle over $Y \times_{X} Y, P$ would be the restriction map $\mathcal{M}\left(V_{Y}\right) \rightarrow \mathcal{M}(V)$ (concerning the continuous moduli) as then $V=\left.V_{Y}\right|_{X}$.
} 
The moduli $v_{i}$ of $V$ are an image of the moduli $c_{j}$ of $\mathcal{M}_{Y}(C)$ under $P$.

This means that when it comes to taking derivatives ${ }^{38}$ of the superpotential $W$ that one has from $\frac{\partial W}{\partial c_{j}}=\sum_{i} \frac{\partial v_{i}}{\partial c_{j}} \frac{\partial W}{\partial v_{i}}$ only an implication in the wrong direction: from having $\partial_{v_{i}} W=0$ to $\partial_{c_{j}} W=0$ instead of the opposite what would be the implication we want; for our goal is the relation $\partial_{v_{i}} W=0$ and where we have control is $\partial_{c_{j}} W=0$. However, the surjection (4.19) tells us in particular that there are 'more' $c_{j}$ moduli than $v_{i}$ moduli. With our control over the $\partial_{c_{j}} W$ we might have enough information to solve our proper problem. Assume a moduli choice $\left\{c_{j}^{*}\right\}$ in a solution set in $\mathcal{M}_{Y}(C)$ solves $\partial_{c_{j}} W=W=0$ and let $\left\{v_{i}^{*}\right\}$ be the image under (4.19) in the image of the solution set; clearly this will also solve $W=0$. In the relation $\left(\partial_{c_{j}} W\right)_{j}=A \cdot\left(\partial_{v_{i}} W\right)_{i}$ the $j$-index runs over $1, \ldots, p$ and the $i$-index over $1, \ldots, q$ where $p \geq q$; so rank $A \leq q$. Choose local coordinates such that only the first $q$ lines of $A$ are non-zero, constituting a matrix $\mathcal{A}$; under the (generic) assumption $\operatorname{rank} \mathcal{A}=q$ we can invert the relation of derivatives and have then the possibility to make an implication in the direction we actually want.

The rest of the argument for having vanishing Pfaffians (at certain moduli), now for all instanton curves in $X$, runs in parallel to the earlier set-up. If one specialises the $c_{j}$-moduli to the set (4.22) below one finds that the Pfaffians for all instanton curves $\mathcal{C}$ in $X$ will vanish at the corresponding $v_{i}$-moduli; again one can include higher multiplicities from tuning $S$ suitably such that $r:=\min _{i} r_{i}$ is suitably large where

$$
\begin{aligned}
r_{i} & :=S \cdot \mathcal{C}_{i} \\
\chi_{i} & :=\lambda \cdot \mathcal{C}_{i}
\end{aligned}
$$

Actually one now is in a set-up with different $\chi_{i}$ for each $i$ (cf. app. A.1 and app. C). Under suitable assumptions, like maximal rank (of the differential) of the mapping $P$, as described above, and for $\lambda$ ample ${ }^{39}$, one finds (cf. app. C for the proof; furthermore here the notation concerning the moduli space has to be read with footn. 28 in mind)

Theorem $P f a f f_{\text {all } c \subset X}$

a) For a generic elliptically embedded projected $S U(n)$ bundle $V$ on an arbitrary CalabiYau threefold $X$ one finds common zeroes for the Pfaffians $P$ faf $f_{\mathcal{C}_{i}}$ of all instanton curves $\mathcal{C}_{i}$ by choosing the bundle moduli from the image under $P$ in (4.19) of the set

$$
\mathcal{R}:=\left\{t \in \mathcal{M}_{Y}(C)\left|a_{n}\right| a_{j} \text { for } j=2, \ldots, n-1\right\}
$$

b) one gets a suitable multiplicity $k \geq 2$ for all $\mathcal{C}_{i}$.

\footnotetext{
${ }^{38}$ we may assume that we pose also the condition $W=0$ bringing us back from covariant derivatives (including the Kahler potential) to ordinary derivatives

${ }^{39}$ and not being even, for simplicity, cf. the remark after (C.1); also, for simplicity, we did assume $h^{1,0}(Y)=0$ and $C$ ample in $Y$
} 


\subsection{Topological vanishing of the Paffian}

For completeness we should point out that here again there will be cases where one gets vanishing results for Pfaffians which are completely independent of the continuous moduli, i.e. where a Pfaffian vanishes along a whole component of the moduli space ('topological vanishing').

To get such results one adopts the same strategy as in sect. 3.5: one searches for the numerical conditions on the parameters $\mu \in \frac{1}{2} \mathbf{Z}$ and $r_{i} \in \mathbf{Z}$ (where now under our assumptions, cf. app. C, one can assume that $r_{i}>n \chi_{i}$ and $\chi_{i}>0$ ) which assure the effectivity of the divisor of the relevant line bundle on the respective $c_{i}$, again already from effectivity of the corresponding divisor on $\mathcal{E}_{i}=\pi^{-1} c_{i}$ (cf. for these divisors the first lines in (C.1)).

We give again the exceptional cases for the cases of interest, i.e. $S U(3), S U(4), S U(5)$ bundles which lead to corresponding GUT groups in the (visible) unbroken gauge group. Theorem 2 Pfaff $f_{\text {all } \mathcal{C} \subset X}$

For a generic elliptically embedded projected $S U(n)$ bundle on an arbitrary Calabi-Yau threefold the Pfaffians $P f a f f_{\mathcal{C}_{i}}$ of all instanton curves $\mathcal{C}_{i} \subset X$ vanish identically in the following cases:

a) First the general cases (independent of the $r_{i}$ ):

$$
\begin{aligned}
& \underline{n \text { odd : }} \mu= \pm 1 / 2 \\
& \underline{n \text { even }:} \mu=0
\end{aligned}
$$

b) Furthermore there are the following exceptional cases (now $\mu>1 / 2$ ):

$S U(2):$ universal

$\underline{S U(3):} 3 \chi_{i}<r_{i} \leq 3 \chi_{i}+\frac{2 \chi_{i}-1}{\mu-\frac{1}{2}}$

$\underline{S U(4):} 4 \chi_{i}<r_{i} \leq 4 \chi_{i}+\frac{\frac{5}{2} \chi_{i}-1}{\mu-\frac{1}{2}}$

$\underline{S U(5):} 5 \chi_{i}<r_{i} \leq 5 \chi_{i}+\frac{3 \chi_{i}-1}{\mu-\frac{1}{2}}$

As in the previous case of such a theorem (for just the base curves of an elliptic Calabi-Yau space) here again in the exceptional cases it will be usually difficult to fulfil the conditions on the $r_{i}$ for all the curves $\mathcal{C}_{i}$. This will be especially so if there are 'many' contributing curves $\mathcal{C}_{i}$; as described in remark b) of sect. 1.2.2, in connection with the notion of generically Kähler-determined bundles, this is usually the interesting regime.

Note that an exceptional case (for $n \neq 2$ ) implies $\mu \leq \frac{n+1}{2} \chi_{\min }-\frac{1}{2}$, which gives, for example, for $\chi_{\min }=1$ that $\mu=\frac{3}{2}$ for $S U(3), 1$ or 2 for $S U(4)$ and $\frac{3}{2}$ or $\frac{5}{2}$ for $S U(5)$. 


\section{Summary and Conlusions}

If one wants to investigate a 'global' string model, with gravity not decoupled and not just a string-motivated field theory, the heterotic string constitutes in many respects still the method of choice (especially in view of the fact that the precise mathematical global geometric study of singular Calabi-Yau fourfolds, whose singularities are just chosen locally in the hope that everything fits nicely together, is still in its infancy). The vast majority of the moduli of such a model is then given by the moduli of the vector bundle, the relevant superpotential being the one generated by world-sheet instantons. Here the conditions $W=D W=0$ for having a supersymmetric Minkowski vacuum are difficult to evaluate if one is not in one of the cases where the sum over all the contributions from the different instanton curves $\mathcal{C}_{i}$ vanishes identically anyway: first one has then to handle the phase factor subtlety to sum up in a reasonable manner the individual contributions, and second it seems difficult to have control over what might then just be 'accidental cancellations' between the individual contributions.

So an interesting way of producing solutions is to try to make vanish all the contributions from the different instanton curves individually (nota bene: for special values of the moduli). Though this may not give all solutions, it may give all which are of a reasonable conceptual structure (given the otherwise accidental nature of the cancellations ${ }^{40}$ ).

The problem with this philosophy is, of course, rooted just in the special type of such solutions: they demand more conditions - individual vanishing (for special values) of the contributions of all $\mathcal{C}_{i}$ - than actually necessary (which would mean that only the sum vanishes). Therefore the following difficulty arises: even if one finds a set of moduli which makes the contribution, actually the Pfaffian $P$ faf $f_{\mathcal{C}_{i}}$, of a specific instanton curve $\mathcal{C}_{i}$ vanish, by no means it can be taken for granted that these sets for all the different instanton curves $\mathcal{C}_{i}$ have a non-empty intersection $\bigcap_{i}\left(P f a f f_{\mathcal{C}_{i}}\right)$. Clearly this should only be the case if the individual conditions (making a single Pfaffian vanish) had already some 'global' origin.

It turns out that precisely such a scenario is realised for spectral cover bundles on elliptically fibered Calabi-Yau spaces $\pi: X \rightarrow B$. However, given the specifics of this construction, although the (sufficient) vanishing conditions, found in [11] and recalled here in Theorem $\operatorname{Pfaf} f_{\text {single }}$, really fit together nicely (i.e. with nonempty intersection) for a large class of contributions, one has in the end control only over the contributions from base instanton curves $\mathcal{C}_{i} \subset B$, what constitutes our Theorem $P$ faf $f_{\text {all } \mathcal{C}_{i} \subset B}$ in sect. 3 .

\footnotetext{
${ }^{40}$ keep in mind that we do not speak of cancellations which happen universally for all moduli - a case for which definitely there are good conceptual reasons [3] - but only for special moduli
} 
Isolating the feature $\left.V\right|_{B} \cong \pi_{C *} L$ (where $C \rightarrow B$ was a surface cover of the base) which made progress possible in this case we define in sect. 4 a corresponding class of 'projected' bundles where just such a property holds over the whole (arbitrary) Calabi-Yau space $X$. To have control over the different threefold covers $C$ of $X$ we assume $C$ embedded in a fourfold $Y$ (not Calabi-Yau). For this class one can try to adopt the analogy with the scenario of spectral bundles over an elliptic $X$ to gain this time control over the contributions from all instanton curves $\mathcal{C}_{i} \subset X$. To have as explicit control as possible over the different covers $C_{3} \subset Y_{4}$ we assume in addition that $Y_{4}$ is fibered over $X_{3}$; for the purpose of proving our main theorem we assume that this fibration is elliptic.

So two things have to be done. First one should see that this class of bundles satisfies the Donaldson-Uhlenbeck-Yau equation $F_{a \bar{b}} g^{a \bar{b}}=0$, i.e. one should make sure that this class contains examples which have a non-empty region of stability in the Kähler moduli space $\mathcal{M}_{K}$. The other thing is, of course, that one should carry out the analogy and prove the relevant theorem that there is a non-empty locus in the moduli space $\mathcal{M}=\mathcal{M}_{V} \times \mathcal{M}_{c x}$ (actually already in $\mathcal{M}_{V}$ ) where the contributions from all Pfaffians $\operatorname{Pfa} f f_{\mathcal{C}_{i}}$ vanish.

We can accomplish both things, though, unfortunately, not at the same time. We realise that spectral cover bundles on an elliptic Calabi-Yau space $X$ constitute an example of our class of elliptically embedded projected bundles ${ }^{41}$. So, as these spectral bundles are known to have a non-empty stability region, our class includes indeed stable bundles.

To accomplish our main goal, to prove the Theorem $P$ faf $f_{\text {all }} \mathcal{C}_{i} \subset X$ which gives an explicit non-empty locus in the bundle moduli space where all individual Pfaffian $P f a f f_{\mathcal{C}_{i}}$ vanish simultaneously, we need an innocent and canonical looking genericity assumption. Unfortunately, this assumption is, however, violated in the spectral case. So, although we reach our main goal to give a theorem which gives vanishing control over all instanton contributions $\operatorname{Pfaf} f_{\mathcal{C}_{i}}$ simultaneously in a class of bundles, the 'elliptically embedded projected' ones, which contains stable bundles, one still would like to see examples where genericity and stability can be made sure at the same time.

Apart from this we feel that this strategy, to find solutions by making vanish the individual terms in the sum over all $\mathcal{C}_{i}$ and finding scenarios where the ensuing conditions fit together 'globally', constitutes a valuable option (if not the only one) to find solutions of conceptual origin in the vast class of interesting bundles where there does not hold a theorem making the sum vanish identically.

\footnotetext{
${ }^{41}$ note that the elliptic fibration aspect in the two members of this assertion refers to different things: first, that $X \rightarrow B$ itself is elliptically fibered, and second that the threefold cover $C_{3}$ lies in an ambient fourfold $Y_{4}$ which itself is elliptically fibered over $X$, i.e. that one has a fibration $Y \rightarrow X$
} 
I thank B. Andreas for discussion. I thank the DFG for support in the project CU 191/1-1 and the SFB 647 and the FU Berlin for hospitality.

\section{A Spectral cover construction}

Compactification on a Calabi-Yau three-fold $X$ with vector bundle $V$ embedded in $E_{8} \times E_{8}$ gives a four-dimensional heterotic string model of $N=1$ supersymmetry. For example, the case of $V$ the tangent bundle leads to an unbroken gauge group $E_{6}$ (times a hidden $\left.E_{8}\right)$. The generalisation to an $S U(n)$ bundle $V_{1}$ gives unbroken GUT groups like $S O(10)$ and $S U(5)$ (we will in the following focus on the visible sector and assume a trivial bundle $V_{2}$ embedded in the second $\left.E_{8}\right)$.

The case where $X$ is elliptically fibered over a base surface $B$ makes possible an explicit description of the bundle by using the spectral cover surface $C$ of $B$, cf. sect. 3 . In this description the $S U(n)$ bundle $V$ is encoded in two data: a class $\eta \in H^{1,1}(B)$ and a class $\gamma \in H^{1,1}(C)$ (the latter is connected to the possible existence of chiral matter in these models [12]). One considers $V$ first over an elliptic fibre $F$ and then globalises over the base $B$. Now, over $F$ the bundle $V$ (assumed to be fibrewise semistable) decomposes as a direct sum of line bundles of degree zero; this is described as a set of $n$ points which sum to zero. If one now lets this vary over the base $B$ it will give a hypersurface $C \subset X$ which is a ramified $n$-fold cover of $B$; we assume its class given by (with pullback understood)

$$
C=n \sigma+\eta
$$

For this one takes $C$ as given as a locus $w=a_{0}+a_{2} x+a_{3} y+\ldots a_{n} x^{\frac{n}{2}}=0$, for $n$ even, say, and $x, y$ the usual elliptic Weierstrass coordinates, with $w$ a section of $\mathcal{O}(\sigma)^{n} \otimes \mathcal{N}$ (here $\mathcal{N}$ is a line bundle of class $\eta$ ); note that $a_{i}$ is of class $\eta-i c_{1}$ (with $c_{1}:=c_{1}(B)$ ).

The idea is then to describe the $S U(n)$ bundle $V$ over $X$ by a line bundle $L$ over $C$

$$
V=p_{*}\left(p_{C}^{*} L \otimes \mathcal{P}\right)
$$

with $p: X \times{ }_{B} C \rightarrow X$ and $p_{C}: X \times{ }_{B} C \rightarrow C$ the projections and $\mathcal{P}$ the global version of the Poincare line bundle over $F \times F$ (actually one uses a symmetrized version of this), i.e. the universal bundle which realizes the second $F$ in the product as the moduli space of degree zero line bundles over the first factor [2].

A second parameter in the description of $V$ is given by a half-integral number $\lambda$ which occurs because one gets from the condition $c_{1}(V)=\pi_{*}\left(c_{1}(L)+\frac{c_{1}(C)-c_{1}}{2}\right)=0$ that with 
$\gamma \in \operatorname{ker} \pi_{*}: H^{1,1}(C) \rightarrow H^{1,1}(B)$ one has

$$
c_{1}(L)=-\frac{1}{2}\left(c_{1}(C)-\pi_{C *} c_{1}\right)+\gamma=\left.\frac{n \sigma+\eta+c_{1}}{2}\right|_{C}+\gamma
$$

where generally one has for the class $\gamma$ just the following possibility

$$
\gamma=\left.\lambda\left(n \sigma-\eta+n c_{1}\right)\right|_{C}
$$

Here $\lambda \in \frac{1}{2} \mathbf{Z}$ and $\lambda$ has to be strictly half-integral for $n$ odd; for $n$ even an integral $\lambda$ needs $\eta \equiv c_{1} \bmod 2$ while a strictly half-integral $\lambda$ needs $c_{1}$ even.

Two conditions on $\eta$ are imposed [9]. First the effectivity of $C$ amounts to $\eta$ being effective. Second to guarantee that $V$ is a stable vector bundle one assumes irreducibility of $C$ (which follows if the linear system $\eta$ is base point ${ }^{42}$ free and $\eta-n c_{1}$ effective). Often one supposes in addition, as we will do also, that $C$ is even ample in $X$ (such that $H^{0,1}(C)=0$ and $L$ is determined by its Chern class); then $\eta-n c_{1}$ is ample in $B$.

For use in sect. 3.5 let us also recall the following facts

$$
\begin{aligned}
& c_{2}(X)=12 c_{1} \sigma+11 c_{1}^{2}+c_{2} \\
& c_{2}(V)=\eta \sigma-\frac{n^{3}-n}{24} c_{1}^{2}+\left(\lambda^{2}-\frac{1}{4}\right) \frac{n}{2} \eta\left(\eta-n c_{1}\right)
\end{aligned}
$$

Assuming ${ }^{43}$ that in the second, hidden $E_{8}$ a trivial bundle is embedded one finds for the fivebrane class $W=W_{B} \sigma+a_{f} F$

$$
W=\left(12 c_{1}-\eta\right) \sigma+11 c_{1}^{2}+c_{2}+\frac{n^{3}-n}{24} c_{1}^{2}-\left(\lambda^{2}-\frac{1}{4}\right) \frac{n}{2} \eta\left(\eta-n c_{1}\right)
$$

Therefore the effectivity demand gives the conditions

$$
\begin{aligned}
\eta & \leq 12 c_{1} \\
\left(\lambda^{2}-\frac{1}{4}\right) \frac{n}{2} \eta\left(\eta-n c_{1}\right) & \leq N_{B}
\end{aligned}
$$

(where $a \leq b$ for classes means that $b-a$ is effective). Here we have defined the bundleindependent number $N_{B}$ (we assume the rank $n$ fixed throughout)

$$
N_{B}=\left(10+\frac{n^{3}-n}{24}\right) c_{1}^{2}+12
$$

using Noether's relation $c_{1}^{2}+c_{2}=12$ for the rational $B$.

\footnotetext{
${ }^{42}$ a base point is a point common to all members of the system; on $B$ a Hirzebruch surface $\mathbf{F}_{\mathbf{k}}$ with base $\mathbf{P}^{\mathbf{1}} b$ and fibre $\mathbf{P}^{\mathbf{1}} f$ this amounts to $\eta \cdot b \geq 0$

${ }^{43}$ to avoid the following restrictions on $\lambda$ one would have to use a hidden bundle of non-effective $c_{2}\left(V_{h i d}\right)$
} 
Let us now invoke the assumption that $C$ is ample and furthermore that $c_{1}$ is effective ${ }^{44}$. Then one finds, as $\eta-n c_{1}$ is ample, that $n \leq n c_{1}\left(\eta-n c_{1}\right)<\eta\left(\eta-n c_{1}\right)$, thus giving

$$
n<\eta\left(\eta-n c_{1}\right) \leq \frac{2 N_{B}}{n\left(\lambda^{2}-\frac{1}{4}\right)}
$$

(for $\lambda \neq \pm 1 / 2,0$ ). This entails in particular the following restriction on $\lambda$

$$
\lambda^{2}-\frac{1}{4}<\frac{2 N_{B}}{n^{2}}
$$

For our main example class of bases $B$ with instanton curves, the del Pezzo surfaces $\mathbf{d P}_{\mathbf{k}}$, $k=1, \ldots, 8$ of $c_{1}^{2}=9-k$, one has $N_{\mathbf{d P}_{\mathbf{k}}} \leq N_{\mathbf{F}_{\mathbf{1}}}=92+\frac{n^{3}-n}{3}$. Thus one has for $S U(3)$ that $\lambda^{2}-\frac{1}{4}<200 / 9$, thus leaving (besides $\pm \lambda=\frac{1}{2}$ ) the possibilities $\pm \lambda=\frac{3}{2}, \frac{5}{2}, \frac{7}{2}$, $\frac{9}{2}$. For $S U(4)$ one gets $\lambda^{2}-\frac{1}{4}<14$, leaving (besides $\lambda=0$ ) the cases $\pm \lambda=1,2,3$. Finally for $S U(5)$ one has $\lambda^{2}-\frac{1}{4}<264 / 25$, thus leaving (besides $\pm \lambda=\frac{1}{2}$ ) the possibilities $\pm \lambda=\frac{3}{2}, \frac{5}{2}$.

\section{A.1 World-sheet instantons contributing to the superpotential}

The (smooth) rational curve $\mathcal{C}$ relevant for the world-sheet instanton which we consider in sect. 3 is a $\mathbf{P}^{\mathbf{1}}$ lying in the base $B$. So, besides the $n: 1$ covering of $B$ by $C$ which lies in the elliptically fibered $X$ threefold over $B$, one has, with $\mathcal{E}:=\pi^{-1} \mathcal{C}$ the elliptic surface over $\mathcal{C}$ and $c:=\left.C\right|_{\mathcal{E}}$, the corresponding spectral curve $n: 1$ covering $\pi_{c}: c \rightarrow \mathcal{C}$ in the elliptic surface $\mathcal{E}$ over $\mathcal{C}$. Now $C=n \sigma+\eta$ gives $c=n s+r F$ with the restrictions $s:=\left.\sigma\right|_{\mathcal{E}}(=\mathcal{C}), r:=\eta \cdot \mathcal{C}$. Note that $r>n c_{1} \cdot \mathcal{C}=n \chi\left(\right.$ cf. below) as $\eta-n c_{1}$ is ample.

Examples are $B=\mathbf{F}_{\mathbf{k}}(k \leq 2)$ where $c_{1}\left(\mathbf{F}_{\mathbf{k}}\right)=2 b+(2+k) f$ with $c_{1} \cdot \mathcal{C}=2-k$ for $\mathcal{C}=b$ (the base $\mathbf{P}^{\mathbf{1}}$ ) or $B=\mathbf{P}^{\mathbf{2}}$ of $c_{1}=3 l$ with $c_{1} \cdot l=3$ and $c_{1} \cdot 2 l=6$ for $\mathcal{C}$ the line and the quadric, respectively. Examples on del Pezzo surfaces are discussed below. Note that $c_{1}(\mathcal{E})=-\pi_{\mathcal{E}}^{*}\left(K_{\mathcal{C}}+\mathcal{O}_{\mathcal{C}}(\chi)\right)=(2-\chi) F$ with $\chi:=\chi\left(\mathcal{E}, \mathcal{O}_{\mathcal{E}}\right)=\frac{1}{12} e(\mathcal{E})=c_{1} \cdot \mathcal{C}$ (as the discriminant of $Z$ over $B$ is given by $\Delta=12 c_{1}$ ), so for the mentioned examples one gets $\mathcal{E}=K 3, \mathbf{d P}_{\mathbf{9}}, b \times F$ for $B=\mathbf{F}_{\mathbf{k}}, \mathcal{C}=b$ with $k=0,1,2$, respectively, and $e(\mathcal{E})=36$ or 72 for the two cases in $\mathbf{P}^{\mathbf{2}}$.

One has $\left.V\right|_{B}=\pi_{C *} L$ and so also $\left.V\right|_{\mathcal{C}}=\left.\pi_{c *} L\right|_{c}$. Now [1] gives $W_{\mathcal{C}} \neq\left. 0 \Leftrightarrow V\right|_{\mathcal{C}}$ trivial or

$$
W_{\mathcal{C}} \neq 0 \Longleftrightarrow 0=h^{0}\left(\mathcal{C},\left.V\right|_{\mathcal{C}} \otimes \mathcal{O}_{\mathcal{C}}(-1)\right)=h^{0}\left(c,\left.L\right|_{c} \otimes \mathcal{O}_{c}\left(-\left.F\right|_{c}\right)\right)
$$

(we will use also the notation $\left.V(-1)\right|_{\mathcal{C}}:=\left.V\right|_{\mathcal{C}} \otimes \mathcal{O}_{\mathcal{C}}(-1)$ and $\left.L(-F)\right|_{c}:=\left.L\right|_{c} \otimes \mathcal{O}_{c}\left(-\left.F\right|_{c}\right)$ ) where one has explicitly

$$
\left.L\right|_{c} \otimes \mathcal{O}_{c}\left(-\left.F\right|_{c}\right)=\left.\mathcal{O}_{\mathcal{E}}\left(n\left(\lambda+\frac{1}{2}\right) s+\left[\left(n \lambda+\frac{1}{2}\right) \chi-\left(\lambda-\frac{1}{2}\right) r-1\right] F\right)\right|_{c}
$$

\footnotetext{
${ }^{44}$ which is the case for $B$ a Hirzebruch surface $\mathbf{F}_{\mathbf{m}}$ or a del Pezzo surface $\mathbf{d P}_{\mathbf{k}}$; the latter are more thoroughly described at the end of the next subsection
} 
Now recall that $N_{X} \mathcal{C}=\mathcal{O}_{\mathcal{C}}(a) \oplus \mathcal{O}_{\mathcal{C}}(-a-2)$ where, say, $a=\mathcal{C}^{2}$, the selfintersection number in $B$, and $-a-2=s^{2}$, the selfintersection number of $\mathcal{C}$, now considered as (the base) curve $s$ in $\mathcal{E}$; note that one has in $\mathcal{E}$ (when suitably interpreted) $-2=K_{s}=$ $s^{2}+K_{\mathcal{E}} \cdot s=s^{2}+\chi-2$, that is $\chi=-s^{2}$.

If we now take also into account that we want to assume that $\mathcal{C}$ is (actually even infinitesimally) isolated one gets $N_{X} \mathcal{C}=\mathcal{O}_{\mathcal{C}}(-1) \oplus \mathcal{O}_{\mathcal{C}}(-1)$ such that ${ }^{45} \chi=-s^{2}=1$. Therefore among the mentioned examples just the base $b$ in $\mathbf{F}_{\mathbf{1}}$ remains. This is just the del Pezzo surface $\mathbf{d P}_{\mathbf{1}}$. So, as we search in particular for (smooth) rational (-1)-curves we have now a closer look on a del Pezzo surface as base and its (-1)-curves.

\section{The del Pezzo surfaces $\mathbf{d P}_{\mathbf{k}}$}

We collect some facts about del Pezzo surfaces which are examples to be used as base surfaces $B$ of elliptic Calabi-Yau threefolds. Their interest in our set-up lies in the fact that they come with many smooth isolated rational curves, often called $(-1)$ - curves from their self-intersection; we will call them instanton curves.

The del Pezzo surface ${ }^{46} \mathbf{d P}_{\mathbf{k}}$ for $k=0, \ldots, 8$ is the blow-up of $\mathbf{P}^{\mathbf{2}}$ at $k$ points (lying suitably general, i.e. no three points lie on a line, no six on a conic). The exceptional curves from these blow-ups are denoted by $E_{i}, i=1, \ldots, k$. The intersection matrix for $H^{1,1}\left(\mathbf{d P}_{\mathbf{k}}\right)$ in the basis $\left(l, E_{1}, \ldots, E_{k}\right)$ with the proper transform $l$ of the line from $\mathbf{P}^{\mathbf{2}}$ is just $\operatorname{Diag}(1,-1, \ldots,-1)$; furthermore $c_{1}\left(\mathbf{d P}_{\mathbf{k}}\right)=3 l-\sum_{i} E_{i}$ such that $c_{1}^{2}\left(\mathbf{d P}_{\mathbf{k}}\right)=9-k$.

There are for $k \geq 2$ further instanton curves on the $\mathbf{d P}_{\mathbf{k}}$ besides the $E_{i}$ themselves. First one has the $\left(\begin{array}{l}k \\ 2\end{array}\right)$ proper transforms of lines in $\mathbf{P}^{\mathbf{2}}$ through two different blow up points; they have in $d P_{k}$ the classes $l-E_{i}-E_{j}$. Then there are in addition for the higher $\mathbf{d P}_{\mathbf{k}}$ of $k \geq 5$ some exceptional configurations: there are $\left(\begin{array}{l}k \\ 5\end{array}\right)$ curves (proper transforms of conics through five of the points) of classes $2 l-\sum_{n=1}^{5} E_{j_{n}}$ (all occurring $E$-indices $j_{n}$ (and $i$ ) from the set $1, \ldots, k$ have to be different; similarly for the cases below); for $k \geq 7$ there are $k \cdot\left(\begin{array}{c}k-1 \\ 6\end{array}\right)$ curves of classes $3 l-2 E_{i}-\sum_{n=1}^{6} E_{j_{n}}$ (these are proper transforms of singular cubics through all the seven points, with a double point at one of them); finally on $\mathbf{d P}_{\mathbf{8}}$ there are even more special curves: 56 curves of class $4 l-2 \sum_{n=1}^{3} E_{j_{n}}-\sum_{m=1}^{5} E_{j_{m}}$, 28 curves of class $5 l-2 \sum_{n=1}^{6} E_{j_{n}}-\sum_{m=1}^{2} E_{j_{m}}$ and 8 curves of class $6 l-3 E_{i}-2 \sum_{n=1}^{7} E_{j_{n}}$.

\footnotetext{
${ }^{45}$ note that $\chi=\mathcal{C} \cdot c_{1} \not \equiv 0 \bmod 2$ excludes the case $c_{1}$ even and so also the case $n$ even with $\lambda \in \frac{1}{2}+\mathbf{Z}$

${ }^{46}$ sometimes the rational elliptic surface " $\mathbf{d P}_{\mathbf{9}}$ " is included which comes with infinitely many $(-1)$ - curves; for other reasons sometimes the Hirzebruch surface $\mathbf{F}_{\mathbf{0}}=\mathbf{P}^{\mathbf{1}} \times \mathbf{P}^{\mathbf{1}}$ is included; note that $\mathbf{d P}_{\mathbf{0}}=\mathbf{P}^{\mathbf{2}}, \mathbf{d} \mathbf{P}_{\mathbf{1}} \cong \mathbf{F}_{\mathbf{1}}, \mathbf{d} \mathbf{P}_{\mathbf{5}} \cong \mathbf{P}^{4}(2,2), \mathbf{d P _ { 6 }} \cong \mathbf{P}^{\mathbf{3}}(3), \mathbf{d P} \mathbf{P}_{\mathbf{7}} \cong \mathbf{P}_{\mathbf{1 , 1 , 1 , 2}}(4), \mathbf{d} \mathbf{P}_{\mathbf{8}} \cong \mathbf{P}_{\mathbf{1 , 1 , 2 , 3}}(6)$
} 


\section{A.2 A technical aside on the spectral cover equation}

Before we come in the app. B to the proof of the fact that a special property of the coefficients of the spectral cover equation leads to zeroes of the Pfaffian we take - for convenience of the reader - the opportunity to make clear a technical point on this equation. The spectral surface $C$ is given by (3.1) with $w \in H^{0}\left(X, \mathcal{O}(\sigma)^{n} \otimes \pi^{*} \mathcal{N}\right)$ where

$$
\begin{array}{ll}
\underline{n=2} & w=a_{0} z+a_{2} x \\
\underline{n=3} & w=a_{0} z+a_{2} x+a_{3} y \\
\underline{n=4} & w=a_{0} z^{2}+a_{2} x z+a_{3} y z+a_{4} x^{2} \\
\underline{n=5} & w=a_{0} z^{2}+a_{2} x z+a_{3} y z+a_{4} x^{2}+a_{5} x y \\
\underline{n=6} & w=a_{0} z^{3}+a_{2} x z^{2}+a_{3} y z^{2}+a_{4} x^{2} z+a_{5} x y z+a_{6} x^{3}
\end{array}
$$

or in general (where $0 \leq i, j$ and $j \leq 1 ; w$ has degree $\left[\frac{n}{2}\right]=\left\{\begin{array}{ll}\frac{n}{2} & n \text { even } \\ \frac{n-1}{2} & n \text { odd }\end{array}\right.$ in $\left.x, y, z\right)$

$$
\begin{aligned}
w= & \sum_{\substack{m=0 \\
2 i+3 j=m \neq 1}}^{n} a_{m} x^{i} y^{j} z^{\left[\frac{n}{2}\right]-(i+j)} \\
= & a_{0} z^{\left[\frac{n}{2}\right]}+a_{2} x z^{\left[\frac{n}{2}\right]-1}+a_{3} y z^{\left[\frac{n}{2}\right]-1}+\ldots \\
& + \begin{cases}a_{n-2} x^{\left[\frac{n}{2}\right]-1} z+a_{n-1} x^{\left[\frac{n}{2}\right]-2} y z+a_{n} x^{\left[\frac{n}{2}\right]} & n \text { even } \\
a_{n-3} x^{\left[\frac{n}{2}\right]-1} z+a_{n-2} x^{\left[\frac{n}{2}\right]-2} y z+a_{n-1} x^{\left[\frac{n}{2}\right]}+a_{n} x^{\left[\frac{n}{2}\right]-1} y & n \text { odd }\end{cases}
\end{aligned}
$$

Now let us consider the situation on a single fibre $F$. For the elliptic curve $F \subset \mathbf{P}_{\mathbf{x}, \mathbf{y}, \mathbf{z}}^{\mathbf{z}}$ given by the Weierstrass equation $z y^{2}=4 x^{3}-g_{2} x z^{2}-g_{3} z^{3}$ (with zero point $p_{0}=(0,1,0)$ ), the divisor $(z)=l \subset \mathbf{P}^{\mathbf{2}}$ becomes $\left.(z)\right|_{F}=3 p_{0}$ on $F$.

$\underline{n \text { even }}$ To encode $n$ points on $F$ one chooses a homogeneous polynomial $w_{n / 2}^{(h o m)}(x, y, z)$ of degree $n / 2$. From its $3 n / 2$ zeroes on $F$ only $n$, say $q_{i}$, carry information as $n / 2$ of them are always at $p_{0}$ : the rewriting $w_{n / 2}^{(h o m)}(x, y, z)=z^{n / 2} w_{n / 2}^{\text {aff }}(x / z, y / z)$ shows $3 n / 2$ zeroes at $p_{0}$ from the $z$-power, and $n$ poles at $p_{0}$ and $n$ zeroes at the $q_{i}$ from the affine factor. ${ }^{47}$ So one gets on $F$ for the divisor of $w^{(h o m)}$ resp. for the divisor of zeroes of $w^{\text {aff }}$

$$
\left(\left.w^{(h o m)}\right|_{F}\right)=\frac{n}{2} p_{0}+\sum^{n} q_{i} \quad, \quad\left(\left.w^{a f f}\right|_{F}\right)_{0}=\sum^{n} q_{i}
$$

$\underline{n \text { odd }}$ Similarly here extracting a factor $z^{\frac{n-1}{2}}$ gives (when $n$ poles at $p_{0}$ of the affine part cancel $n$ zeroes of the $3 \cdot \frac{n-1}{2}$ zeroes of $\left.z^{\frac{n-1}{2}}\right|_{F}$ )

$$
\left(\left.w^{(h o m)}\right|_{F}\right)=\frac{n-3}{2} p_{0}+\sum^{n} q_{i} \quad, \quad\left(\left.w^{a f f}\right|_{F}\right)_{0}=\sum^{n} q_{i}
$$

\footnotetext{
${ }^{47} x / z$ and $y / z$ have a pole at $p_{0}$ of order $-(1-3)=2$ and $-(0-3)=3$, resp.
} 
Now two cases deserve special attention here. First one may ask: when is at least one of the $n$ zeroes $q_{i}$ the point $p_{0}$ ? Considered in $X$ (or in $\mathcal{E}_{i}$ ) this is, of course, the question at which points $C$ intersects $B$ (or $c_{i}$ intersects $\mathcal{C}_{i}$ ). The answer, involving the zero divisor $\left(a_{n}\right)$, was given (in a reasoning in affine coordinates) in equ. (6.8) in [2]

$$
B \cap C=\left(a_{n}\right) \quad\left(\text { or } \mathcal{C}_{i} \cap c_{i}=\left(a_{n ; i}\right)\right)
$$

Of course, this is also seen in the factorization of the homogeneous polynomial above: if one is at a point $b \in B$ (or $b \in \mathcal{C}_{i}$ ) where the highest coefficient of the spectral cover equation vanishes, then the affine factor $\left.w^{a f f}\right|_{F_{b}}$ has only $n-1$ zeroes $q_{i}$ and $n-1$ poles at $p_{0}$ such that one zero less of the $z$-power $\left.z^{\left[\frac{n}{2}\right]}\right|_{F_{b}}$ is cancelled, giving the net effect that one of the previous (generic) $n$ zeroes $q_{i}$ is now at $p_{0}$. Conversely if one of the $q_{i}$ is at $p_{0}$ it cancels one of the poles there; but as the number of poles is manifest in the occurring highest power this means that its coefficient vanishes.

A second case is decisive for the theorems in the present paper, cf. app. B. This is the case when now not just one of the $n$ points $q_{i}$ on a fibre (encoded by the spectral cover equation) lies actually at $p_{0}$ but when all $n$ zeroes $q_{i}$ are at $p_{0}$. From what was said a moment ago in the first case it is clear that this will just happen if all $n$ poles of the affine factor drop out. This in turn will just happen if all coefficients higher than $a_{0}$ vanish. As we have seen, that ${ }^{48}$ at all points $u_{j ; i}$ of $\left(a_{n}\right) \cap \mathcal{C}_{i}=\left(a_{n ; i}\right) \subset \mathcal{C}_{i}$ already one of the spectral points (the zeroes $q_{i}$ ) is at $p_{0}$, the condition that at these points $u_{j ; i}$ the coefficients $a_{2 ; i}, \ldots, a_{n-1 ; i}$ vanish as well is just given by $a_{n ; i} \mid a_{j ; i}$ for all $j=2, \ldots, n-1$.

This is just the relation $\mathcal{R}_{i} \subset \underline{\Sigma_{i}}$ (actually " =") in app. A.3 which we recall in the proof of assertion a) of Theorem $\operatorname{Pfaf} f_{\text {single } \mathcal{C} \subset B}$ in sect. B below: along this locus $\mathcal{R}_{i}$ (of expected codimension $(n-2)\left(r_{i}-n\right)$ ) in moduli space $\mathcal{M}_{\mathcal{E}_{i}}\left(c_{i}\right)$ the $n$ fibre points of $c_{i}$ over each of the zeroes $u_{j ; i}$ of $a_{n ; i}$ on $\mathcal{C}_{i} \cong \mathbf{P}^{\mathbf{1}}$ will all be just $p_{0}$ because now not only $a_{n ; i}$ vanishes at these points of $\mathcal{C}_{i}$ but actually all the $a_{n ; j}, j=2, \ldots, n-1$, as well.

Remark: One realises $\left(r_{i}-n\right)(n-2)$ (cf. the interpretation of this number in app. A.4) as the codimension of this specialisation locus in $\mathcal{M}_{\mathcal{E}_{i}}\left(c_{i}\right)$ by computing for $h^{0}\left(\mathcal{E}_{i}, \mathcal{O}_{\mathcal{E}_{i}}\left(c_{i}\right)\right)$

$$
h^{0}\left(\mathcal{C}_{i}, \mathcal{O}_{\mathcal{C}_{i}}\left(r_{i}\right)\right)+\sum_{j=2}^{n-1} h^{0}\left(\mathcal{C}_{i}, \mathcal{O}_{\mathcal{C}_{i}}\left(r_{i}-j\right)\right)+h^{0}\left(\mathcal{C}_{i}, \mathcal{O}_{\mathcal{C}_{i}}\left(r_{i}-n\right)\right)=n r_{i}-\frac{n^{2}-n}{2}
$$

whereas ${ }^{49}$ one gets for the degrees of freedom in the specialisation $\mathcal{R}_{i}$

$h^{0}\left(\mathcal{C}_{i}, \mathcal{O}_{\mathcal{C}_{i}}\left(r_{i}\right)\right)+\sum_{j=2}^{n-1} h^{0}\left(\mathcal{C}_{i}, \mathcal{O}_{\mathcal{C}_{i}}(n-j)\right)+h^{0}\left(\mathcal{C}_{i}, \mathcal{O}_{\mathcal{C}_{i}}\left(r_{i}-n\right)\right)=2 r_{i}+\frac{n^{2}-3 n}{2}$

\footnotetext{
${ }^{48}$ now we describe the situation in the set-up $c_{i} \subset \mathcal{E}_{i}$ over $\mathcal{C}_{i}$

${ }^{49} \mathrm{cf}$. with regard to this comparison also the remark a) in sect. 3.4
} 


\section{A.3 Characterisation of the solution locus}

Let us try to understand more fully the conditions in the sets $\mathcal{R}$ and $\Sigma$, or their respective local versions $\mathcal{R}_{i}$ and $\Sigma_{i}$ over an individual instanton curve $\mathcal{C}_{i}$ in $B$, and along this way provide a proof to the proposition stated in sect. (3.4). First one realises that here naturally a further locus $\underline{\Sigma}$ in the moduli space occurs with the following 'logical position'

$$
\mathcal{R} \subset \underline{\Sigma} \subset \Sigma
$$

For this let us first define a corresponding locus $\underline{\Sigma_{i}}$ in the situation for the Pfaffian for one individual instanton curve (we use the zeroes $u_{k ; i}$ of $a_{n ; i}$ where $k$ runs from 1 to $r_{i}-n$; $\left.s_{i}\right|_{c_{i}}$ is in each of the fibers just the respective point $p_{0}$; here $\sim$ is linear equivalence)

$$
\begin{aligned}
\underline{\Sigma_{i}} & :=\left\{t \in \mathcal{M}_{\mathcal{E}_{i}}\left(c_{i}\right)\left|n s_{i}\right|_{c_{i}}=\left.\sum_{k=1}^{r_{i}-n} F_{u_{k ; i}}\right|_{c_{i}}\right\} \\
\Sigma_{i} & =\left\{\left.t \in \mathcal{M}_{\mathcal{E}_{i}}\left(c_{i}\right)\left|n s_{i}\right|_{c_{i}} \sim\left(r_{i}-n\right) F\right|_{c_{i}}\right\}
\end{aligned}
$$

(here for comparison we also recalled an explicit form of the condition defining $\Sigma_{i}$ ). Now we enlarge the consideration from one instanton curve $\mathcal{C}_{i}$ to $B$ as a whole and define correspondingly, in analogy to (A.23), using the curve $A:=\sigma \cap C=\left(a_{n}\right)$,

$$
\underline{\Sigma}:=\left\{t \in \mathcal{M}_{X}(C)|n \sigma|_{C}=\left.\pi^{-1}(A)\right|_{C}\right\}
$$

Now the strategy for proving the mentioned proposition of sect. (3.4) consists in two parts. First one realises that one has directly the inclusions $\mathcal{R}_{i} \subset \underline{\Sigma_{i}} \subset \Sigma_{i}$, respectively $\mathcal{R} \subset \Sigma \subset \Sigma$. Then we will show the opposite inclusions; actually we proceed by treating these reasonings first for the first inclusion (showing that it is actually an equality) and then, in a more extended argument, for the second inclusion (although all of this is completely elementary we give the arguments in detail).

So note first that not just the obvious (cf. app. A.2 and app. B) relations $\mathcal{R}_{i} \subset \underline{\Sigma_{i}}$ and $\mathcal{R} \subset \underline{\Sigma}$ hold but that one actually has $\mathcal{R}_{i}=\underline{\Sigma_{i}}$ and $\mathcal{R}=\underline{\Sigma}$. The first equality is explained in app. A.2. The global version $\mathcal{R}=\underline{\Sigma}$ follows from the same reasoning as the property of a $C$-fibre $\left.F_{b}\right|_{C}$ (over a point $b \in B$ ) to have all its $n$ points at $p_{0}=F_{b} \cap \sigma$ is shown in app. A.2 to be equivalent to the vanishing of $a_{j}(b)$ for $j=2, \ldots, n$. Only the relation $\Sigma \subset \mathcal{R}$ remains to be seen. But the condition $\left.a_{j}\right|_{A}=0$ for $j=2, \ldots, n-1$ indeed entails $a_{j}=a_{j}^{\prime} \cdot a_{n}$ as the chain of $H^{0}$-terms in the long exact sequence associated to the restriction to $A$ (of the respective bundle of which an $a_{j}$ is a section) shows.

Note further that also not just the obvious relations $\underline{\Sigma_{i}} \subset \Sigma_{i}$ and $\underline{\Sigma} \subset \Sigma$ hold but that one actually has $\underline{\Sigma_{i}}=\Sigma_{i}$ and $\underline{\Sigma}=\Sigma$. Though elementary let us give the reasoning in 
detail. As $\left.n s_{i}\right|_{c_{i}}$ is effective there exists a nonzero section $f \in H^{0}\left(c,\left.\mathcal{O}_{\mathcal{E}_{i}}\left(n s_{i}\right)\right|_{c_{i}}\right)$ which fulfils of course, as any such section, the relation $\left.(f) \sim n s_{i}\right|_{c_{i}}$. On the other hand there exists a specific meromorphic section $g$ of the bundle in question with $(g)=\left.n s_{i}\right|_{c_{i}}$; as the latter divisor is effective $g$ is actually a holomorphic section, i.e. an element of $H^{0}$; so we can take an $f$ as before with

$$
(f)=\left.n s_{i}\right|_{c_{i}}
$$

Now, for a modulus $t \in \Sigma_{i}$ for which $\left.\mathcal{O}_{\mathcal{E}_{i}}\left(n s_{i}-\left(r_{i}-n\right) F\right)\right|_{c_{i}}$ becomes trivial there exists a nonzero holomorphic section $\zeta$ of this bundle on the curve $c_{i}$ (as the line bundle in question is flat the existence of such a $\zeta$ is even equivalent for the bundle being trivial: the linearly equivalent effective divisor of $\zeta$ also has degree zero and thus is zero). Clearly, as the line bundle is trivial, $\zeta$ has neither zeroes (nor poles). In other words the element $f / \zeta$ is a nonzero section of $\left.\mathcal{O}_{\mathcal{E}_{i}}\left(\left(r_{i}-n\right) F\right)\right|_{c_{i}}$

$$
\frac{f}{\zeta} \in H^{0}\left(c_{i},\left.\mathcal{O}_{\mathcal{E}_{i}}\left(\left(r_{i}-n\right) F\right)\right|_{c_{i}}\right)
$$

which still has divisor

$$
\left(\frac{f}{\zeta}\right)=\left.n s_{i}\right|_{c_{i}}
$$

At this point a priori one knows only that $\left.\left(\frac{f}{\zeta}\right) \sim\left(r_{i}-n\right) F\right|_{c_{i}}$. But it turns out that now one has actually (where the $b_{k}$ must be the points of $s_{i} \cap c_{i}$ )

$$
\left(\frac{f}{\zeta}\right)=\left.\sum_{k=1}^{r_{i}-n} F_{b_{k}}\right|_{c_{i}}
$$

For this one may note that any relation $\left.D \sim F\right|_{c_{i}}$ entails $D=\left.F_{b_{*}}\right|_{c_{i}}$ as the linear system $|F|_{c_{i}} \mid$ is complete, i.e. contains any effective divisor which is linearly equivalent to any of its members and these members turn out to be just all the $\left.F_{b}\right|_{c_{i}}$ (the corresponding property holds of course for the linear system $|F|$ on $\mathcal{E}_{i}$ ). This is easily inductively generalised for the corresponding relevant property for a multiple fibre class, thereby proving (A.29). ${ }^{50}$ Then (A.28) and (A.29) prove that $\Sigma_{i} \subset \underline{\Sigma_{i}}$.

The argument for the global case $\Sigma \subset \underline{\Sigma}$ is similar: one finds the divisor $(f / \zeta)=\left.n \sigma\right|_{C}$ which is $\sim \pi^{-1} A$ and is actually $=\pi^{-1} A^{\prime}$ with $A^{\prime} \in|A|$ where the $A^{\prime}$ must be $\sigma \cap C=A$.

\footnotetext{
${ }^{50} \mathrm{An}$ alternative reasoning might use the fibration structure instead as follows: from the long exact sequence associated to the short exact sequence involving the restriction from $\mathcal{E}_{i}$ to $c_{i}$ for the bundle $\mathcal{O}_{\mathcal{E}_{i}}\left(\left(r_{i}-n\right) F\right)$ one finds that $H^{0}\left(c_{i},\left.\mathcal{O}_{\mathcal{E}_{i}}\left(\left(r_{i}-n\right) F\right)\right|_{c_{i}}\right) \cong H^{0}\left(\mathcal{E}_{i}, \mathcal{O}_{\mathcal{E}_{i}}\left(\left(r_{i}-n\right) F\right)\right)$, the latter being in turn equal to $\pi^{*} H^{0}\left(\mathcal{C}_{i}, \mathcal{O}_{\mathcal{C}_{i}}\left(r_{i}-n\right)\right)$; this realises $f / \zeta$ as a pullback polynomial $\pi^{*} P_{r_{i}-n}$ restricted to $c_{i}$ which itself has the sought-after divisor.
} 
Thus in total we have by now shown that one has

$$
\begin{gathered}
\mathcal{R}_{i}=\underline{\Sigma_{i}}=\Sigma_{i} \\
\mathcal{R}=\underline{\Sigma}=\Sigma
\end{gathered}
$$

Thereby one has proven the statements a) and b) of the proposition in sect. 3.4.

Remark: This may be the right place to point yet to a further interpretation of the specialisation condition which defines our solution locus $\Sigma_{i}$ or $\Sigma$ in moduli space. It is related directly to the degenerate structure for the spectral cover $C$ which the conditions impose.

As along $\underline{\Sigma}$ (in moduli space) the behaviour of $C$, or $V$, along $A$ (in $X$ ) is decisive let us describe the effect of the spectral degeneration there further (for more on this cf. sect. 3.7). The right hand side in (A.23) can be written also as follows: the locus on $s_{i}$ given by the zero divisor of $a_{n ; i}$ is just $s_{i} \cap c_{i}$, and $c_{i}$ is everywhere over $s_{i}$ just an $n$-fold collection of points in the fibres $F$ over $s_{i}$; so one can write as characterising condition for the set $\underline{\Sigma_{i}}$ equivalently

$$
\underline{\Sigma_{i}}=\left\{t \in \mathcal{M}_{\mathcal{E}_{i}}\left(c_{i}\right)\left|n s_{i}\right|_{s_{i} \cap c_{i}}=\left.c_{i}\right|_{\pi^{-1}\left(s_{i} \cap c_{i}\right)}\right\}
$$

This is in the situation for one instanton curve in the base. Now going back to $B$ as a whole one has correspondingly, in analogy to (A.32), ${ }^{51}$

$$
\underline{\Sigma}=\left\{t \in \mathcal{M}_{X}(C)|n \sigma|_{A}=\left.C\right|_{\pi^{-1}(A)}\right\}
$$

Now, an $S U(m)$ bundle on $X$ which arises as pullback $\pi^{*} M$ of an $S U(m)$ bundle $M$ on the base $B$ has the degenerate spectral data $(m \sigma, M)$, i.e. its spectral cover surface $C$ is the nonreduced object given by taking $B$ with multiplicity $m$. Although we expect a line bundle on the spectral cover surface and not a rank $m$ bundle, here the rank $m$ bundle $M$ (which splits, of course, as a direct sum of $m$ one-dimensional spaces over each point of $B$ ) is understood as a deformation of a line bundle over $m \sigma$.

Now we see that - along the locus $\underline{\Sigma}$ in moduli space - the part $\left.C\right|_{\pi^{-1}(A)}$ of the spectral cover surface $C$ which lies over $A$ has according to (A.25) just such a nonreduced structure. So, over this subset $A$ the role of $M$ is played here by $\left.\left.\left.L\right|_{A} \oplus L\right|_{A} \oplus \ldots \oplus L\right|_{A}=\left.\oplus_{1}^{n} L\right|_{A}$. So, from the previous paragraph, $V$ has now along $A$ also the further ${ }^{52}$ representation $\left.\left.V\right|_{A} \cong\left(\left.\pi^{*} L\right|_{A}\right)\right|_{n A}$, or rather one gets that along $\underline{\Sigma}$ one has

$$
\left.\left.V\right|_{A} \cong \oplus^{n} L\right|_{A}
$$

This is another manifestation of the degenerate structure the conditions impose.

\footnotetext{
${ }^{51}$ in (A.32) one may write $n\left(s_{i} \cap c_{i}\right)$ for $\left.n s_{i}\right|_{s_{i} \cap c_{i}}$, and in (A.25) similarly $n A$ for $\left.n \sigma\right|_{A}$

${ }^{52}$ note that in any case $V$ has along $B=\sigma$ the representation $\left.V\right|_{B} \cong \pi_{C *} L$ and has therefore along $A$ the representation $\left.\left.V\right|_{A} \cong \pi_{\left(C \cap \pi^{-1}(A)\right) *} L\right|_{\pi^{-1}(A)}$
} 


\section{A.4 Conceptual argument for the main implication}

The source from which everything flows in this note is Theorem $\operatorname{Pfaff} f_{\operatorname{single}} \mathcal{C} \subset B$, more precisely the principle of its proof. This is generalised to include the cases of all base curves for a spectral bundle on an elliptic $X$ and then in sect. 4 to the case of certain projected bundles over an arbitrary $X$. We give the proof of this basic assertion in app. B and did recall the idea already in app.A.2. The reasoning relies on the use of the concrete spectral equation for the cover object (the curve $c_{i} \subset \mathcal{E}_{i}$ over $\mathcal{C}_{i}$ ). As this explicit description may be not available in related set-ups it clearly would be of interest to have a more conceptual understanding of the argument which is completely independent of the use of a concrete defining equation of the cover object. Here we will keep the concrete structure of our case but sketch an argument not using the equation, and so in particular also not using the representation of the moduli by the coefficients polynomials $a_{n ; j}$.

So let us consider in the elliptic surface $\mathcal{E}_{i}$ the cover curve $c_{i}$ of class $n s_{i}+r_{i} F$ together with the relevant line bundle $\mathcal{O}_{\mathcal{E}_{i}}\left(n s_{i}-\left(r_{i}-n\right) F\right)$. Its restriction to $c_{i}$ has degree zero and the issue at stake is that the part $-\left(r_{i}-n\right) F$ of the divisor violates manifestly the effectivity on $\mathcal{E}_{i}$. One wants now to show that a concrete curve $c_{i}$, i.e. a member in the linear system $\left|c_{i}\right|$ of curves in $\mathcal{E}_{i}$, exists for which $\left.n s_{i}\right|_{c_{i}}$ and $\left.\left(r_{i}-n\right) F\right|_{c_{i}}$ are linearly equivalent. We show that a member exists for which these divisors on $c_{i}$ are even equal.

First (concerning the zeroes $u_{j} \in \mathcal{C}_{i}=s_{i}$ of $a_{n ; i}$ ) note that there exist $r_{i}-n$ concrete fibers $\left.F_{j}\right|_{c_{i}}$, i.e. members of the linear system $|F|_{c_{i}} \mid$, which contain the zero point $p_{0}$ of the respective fibre (as $\left.s_{i}\right|_{c_{i}}$ has $s_{i} \cdot c_{i}=r_{i}-n$ points $u_{j}$ and $|F|_{c_{i}} \mid$ covers the whole $c_{i}$ ).

Secondly (replacing the argument involving the condition $a_{n ; i} \mid a_{j ; i}$ ) we ask for the existence of a concrete $c_{i}$ (a member of $\left|c_{i}\right|$ ) with $\left.\sum_{j=1}^{r_{i}-n} F_{j}\right|_{c_{i}}=\left.n s_{i}\right|_{c_{i}}$ (the sum taken over the concrete fibers of the first step) ${ }^{53}$; to specialise $n-2$ points to $p_{0}$ in each of the $r_{i}-n$ fibers amounts to $\left(r_{i}-n\right)(n-2)$ conditions $^{54}$. Now the 'restriction' map ${ }^{55}$ (for $k \geq 0$ )

$$
H^{0}\left(\mathcal{E}_{i}, \mathcal{O}_{\mathcal{E}_{i}}\left(c_{i}\right)\right) \rightarrow \bigoplus_{u_{j} \in s_{i} \mid c_{i}} H^{0}\left(u_{j}, \mathcal{O}_{u_{j}} / I_{k+1}^{\left(u_{j}\right)}\right) \cong \bigoplus^{r_{i}-n} \bigoplus \mathbf{C}
$$

(after shown to be surjective) has a kernel of dimension $n r_{i}-\frac{n^{2}-n}{2}+1-k\left(r_{i}-n\right)$ which is $(1+)$ the dimension of the linear subsystem of $\left|c_{i}\right|$ of curves containing $\left.s_{i}\right|_{c_{i}}$ even with multiplicity $k+1$ (and not just the automatic multiplicity 1 ); $k=n-2$ gives the assertion.

\footnotetext{
${ }^{53}$ note that if one of these fibers contains in addition to $p_{0}$ further $n-2$ times the point $p_{0}$ then already all $n$ fibre points are $p_{0}$ as they have to sum up to zero (represented by $p_{0}$ ) in the group law on the fibre

${ }^{54}$ it will then turn out that each of these counts just as one codimension (cf. the remark in app. A.2)

${ }^{55}$ think of the evaluation (A.35) as giving the value and the first $k$ derivatives (at the $r_{i}-n$ points $u_{j}$ of $\left.s_{i}\right|_{c_{i}}$ ) of a 'function' with zero set $c_{i}$; clearly all the values (0'th order derivatives) vanish already
} 


\section{B Proof of Theorem $\operatorname{Pfaff} f_{\operatorname{single} \mathcal{C} \subset B}$}

We recall the proof of parts a) and c) of Theorem $\operatorname{Pfaf} f_{\text {single } \mathcal{C} \subset B}$ from sect. 3.4 (for the first step here, the proof of a), cf. also the reasoning in app. A.2 and A.4).

a) $\underline{\mathcal{R}_{i} \subset \Sigma_{i}}$ : In view of the equation ${ }^{56}$ (3.1) for $C$, or the corresponding ensuing equation $^{57} w_{i}:=a_{0 ; i} z^{n / 2}+a_{2 ; i} x z^{n / 2-1}+a_{3 ; i} y z^{n / 2-1}+\ldots+a_{n ; i} x^{n / 2}=0$, one finds along the locus $\mathcal{R}_{i}$ in moduli space a special coincidence of two in general different divisors on $c_{i}$ : first one has the divisor $\left.s_{i}\right|_{c_{i}}$ which consists of $s_{i} \cdot\left(n s_{i}+r_{i} F\right)=r_{i}-n$ points (counted with multiplicities) which, according to equ. (A.19), lie in fibers $\left.F_{u_{k}}\right|_{c_{i}}$ of $c_{i}$ over the $\operatorname{deg} a_{n ; i}=r_{i}-n$ zeroes $u_{k}$ of the homogeneous polynomial $a_{n ; i}$ on the $\mathbf{P}^{\mathbf{1}}$ given by ${ }^{58}$ $s_{i}$. On the other hand clearly any of these fibers will consist of $n$ points of $c_{i}$. It would be a rather special circumstance if the corresponding $n$ points in each of the $r_{i}-n$ fibers $\left.F_{u_{k}}\right|_{c_{i}}$ over the zeroes of $a_{n ; i}$ would just consist of the intersection point (of coordinates $(x, y, z)=(0,1,0)$ in the $\mathbf{P}^{\mathbf{2}}$ coordinates $)$ of $c_{i}$ with the section $s_{i}$ counted $n$ times. But this is just what happens along the locus $\mathcal{R}_{i}$ as there (cf. sect. A.2), to be in one of the fibers $F_{u_{k}}$, implies $z=0$, i.e. to be on the section $s_{i}$. Therefore the divisors $\left.n s_{i}\right|_{c_{i}}$ and $\left.\left.\sum_{k=1}^{r_{i}-n} F_{u_{k}}\right|_{c_{i}} \sim\left(r_{i}-n\right) F\right|_{c_{i}}$ (where $\sim$ is linear equivalence) are the same, and so the line bundle on $c_{i}$ corresponding to the difference of these divisors is trivial indeed.

$\underline{\Sigma_{i} \subset P f a f f_{\mathcal{C}_{i}}}$ : Because of $h^{0}\left(\mathcal{C}_{i},\left.V\right|_{\mathcal{C}_{i}} \otimes \mathcal{O}_{\mathcal{C}_{i}}(-1)\right)=h^{0}\left(c_{i},\left.L\right|_{c_{i}} \otimes \mathcal{O}_{c_{i}}\left(-\left.F\right|_{c_{i}}\right)\right)$ one needs to find a nontrivial section of $\left.L\right|_{c_{i}} \otimes \mathcal{O}_{c_{i}}\left(-\left.F\right|_{c_{i}}\right)$. Now, concretely one has with (A.14)

$$
\begin{aligned}
\left.L\right|_{c_{i}} \otimes \mathcal{O}_{c_{i}}\left(-\left.F\right|_{c_{i}}\right)= & \begin{cases}\left.\mathcal{O}_{\mathcal{E}_{i}}\left(\frac{1}{2}\left[n s_{i}+\left(r_{i}-1\right) F\right]+\lambda\left[n s_{i}-\left(r_{i}-n\right) F\right]\right)\right|_{c_{i}} & \text { for } n \text { even } \\
\left.\mathcal{O}_{\mathcal{E}_{i}}\left(\left(\lambda+\frac{1}{2}\right) n s_{i}+\left[\left(\lambda n-\frac{1}{2}\right)-\left(\lambda-\frac{1}{2}\right) r_{i}\right] F\right)\right|_{c_{i}} & \text { for } n \text { odd }\end{cases} \\
& \stackrel{\Sigma_{i}}{\rightarrow}\left\{\begin{array}{l}
\left.\mathcal{O}_{\mathcal{E}_{i}}\left(\frac{n}{2} s_{i}+\frac{r_{i}-1}{2} F\right)\right|_{c_{i}} \\
\left.\mathcal{O}_{\mathcal{E}_{i}}\left(\left[r_{i}-\frac{n+1}{2}\right] F\right)\right|_{c_{i}}
\end{array}\right.
\end{aligned}
$$

(to use $\left.\left.n s_{i}\right|_{c_{i}} \sim\left(r_{i}-n\right) F\right|_{c_{i}}$ we write first different forms of the same expression because of the half-integrality issue; note that for $n$ even one has $\lambda \in \mathbf{Z}$ and $r_{i}$ odd, while for $n$ odd one has $\lambda \in \frac{1}{2}+\mathbf{Z}$ ). (B.1) shows the effectivity of the relevant divisors along $\Sigma_{i}$.

c) First one gets, in refinement of the criterion $\operatorname{Pfaf} f_{\mathcal{C}}(t)=0 \Longleftrightarrow h^{0}\left(c_{t},\left.L(-F)\right|_{c_{t}}\right) \neq$ 0 , that the vanishing order of $P f a f f_{\mathcal{C}}(t)$ is bounded below by $h^{0}\left(c_{t},\left.L(-F)\right|_{c_{t}}\right)$ (cf. (4.38) in [11]). From the long exact sequence one finds that the sections of the corresponding bundles on $\mathcal{E}$ in (B.1) inject into the sections of the bundles restricted to $c_{t}$. This

\footnotetext{
${ }^{56}$ written in affine coordinates $(x, y)$ of the $\mathbf{P}^{\mathbf{2}}$ fibre

${ }^{57}$ for $n$ even, say; now written in projective coordinates $(x, y, z)$; note the subtleties (A.17),(A.18)

${ }^{58} s_{i}$ is the base (and section) of the elliptic surface $\mathcal{E}_{i}:=\pi^{-1}\left(\mathcal{C}_{i}\right)$ which shares the general elliptic fibre $F$ with $X$; that is, $s_{i}$ (which is also $\sigma \cap \mathcal{E}_{i}$ ) is the curve $\mathcal{C}_{i}$ considered in $\mathcal{E}_{i}$ instead of $B$
} 
gives the assertion when using (for $n$ even; note $r>n$ ) that $h^{0}\left(\mathcal{E}, \mathcal{O}_{\mathcal{E}}\left(\frac{n}{2} s+\frac{r-1}{2} F\right)\right)=$ $h^{0}\left(\mathcal{C}, \mathcal{O}_{\mathcal{C}}\left(\frac{r-1}{2}\right) \oplus \bigoplus_{i=2}^{n / 2} \mathcal{O}_{\mathcal{C}}\left(\frac{r-1}{2}-i\right)\right)=\frac{r-1}{2}+1+\sum_{i=2}^{n / 2}\left(\frac{r-1}{2}-i+1\right)=\frac{n}{2}\left(\frac{r-1}{2}+1\right)-\left(\frac{1}{2} \frac{n}{2}\left(\frac{n}{2}+1\right)-1\right)$.

\section{Deviations in the proof of theorem $\operatorname{Pfaf} f_{\text {all } C \subset X}$}

The essential deviation in the set-up where we have an elliptically fibered fourfold $Y$ (not being a Calabi-Yau space) over our arbitrary Calabi-Yau threefold $X$ lies in the fact that now no longer the isolatedness of an instanton curve $\mathcal{C}_{i}$ in $X$ is related to the value of $\chi_{i}:=\mathcal{C}_{i} \cdot \lambda$ where $\lambda=c_{1}(\mathcal{L}) \in H^{2}(X)$ encodes the fibration (cf. (4.14); previously this was the class $c_{1}=c_{1}(B) \in H^{2}(B)$, and we found $\chi_{i}=1$ in app. A.1).

Having now many individual $\chi_{i}$ (which is still $-s_{i}^{2}$, but $\mathcal{E}_{i}=\pi^{-1}\left(\mathcal{C}_{i}\right)$ will be no longer $\mathbf{d P}_{\mathbf{9}}$ in general), let us see how the arguments in the proof of Theorem $P$ faf $f_{\text {single } C \subset B}$ have to be adapted. We assume $\lambda$ and $C$ (so also $S-n \lambda$ ) ample, so $\chi_{i}>0$ and $r_{i}>n \chi_{i}$.

a) $\underline{\mathcal{R}_{i} \subset \Sigma_{i}}$ : Here one finds, with $c_{i}=n s_{i}+r_{i} F$, again that $\left.s_{i}\right|_{c_{i}}$ consists of $r_{i}-n \chi_{i}$ points and gets the crucial relation of divisors $\left.n s_{i}\right|_{c_{i}}=\left.\left.\sum_{k=1}^{r_{i}-n \chi_{i}} F_{u_{k}}\right|_{c_{i}} \sim\left(r_{i}-n \chi_{i}\right) F\right|_{c_{i}}$.

$\underline{\Sigma_{i} \subset \operatorname{Pfaf} f_{\mathcal{C}_{i}}}$ : Here one finds the following (to avoid notational confusion we have denoted the twist parameter in this set-up by $\mu$; for $\chi_{i}=1$ everything reduces to (B.1))

$$
\begin{aligned}
\left.L\right|_{c_{i}} \otimes \mathcal{O}_{c_{i}}\left(-\left.F\right|_{c_{i}}\right) & = \begin{cases}\left.\mathcal{O}_{\mathcal{E}_{i}}\left(\frac{1}{2}\left[n s_{i}+\left(r_{i}+\chi_{i}-2\right) F\right]+\mu\left[n s_{i}-\left(r_{i}-n \chi_{i}\right) F\right]\right)\right|_{c_{i}} & \text { for } n \text { even } \\
\left.\mathcal{O}_{\mathcal{E}_{i}}\left(\left(\mu+\frac{1}{2}\right) n s_{i}+\left[\left(\mu n \chi_{i}+\frac{\chi_{i}-2}{2}\right)-\left(\mu-\frac{1}{2}\right) r_{i}\right] F\right)\right|_{c_{i}} & \text { for } n \text { odd }\end{cases} \\
& \stackrel{\Sigma_{j}}{\rightarrow}\left\{\begin{array}{l}
\left.\mathcal{O}_{\mathcal{E}_{i}}\left(\frac{n}{2} s_{i}+\frac{r_{i}+\chi_{i}-2}{2} F\right)\right|_{c_{i}} \\
\left.\mathcal{O}_{\mathcal{E}_{i}}\left(\left[r_{i}-\frac{(n-1) \chi_{i}+2}{2}\right] F\right)\right|_{c_{i}}
\end{array}\right.
\end{aligned}
$$

(here one may compare directly with (A.3) and (A.4); we did assume here that the case $\lambda$ even does not occur, so $n$ even does not allow for a strictly half-integral $\mu$; in the former set-up this assumption (then: $c_{1}$ not even) was fulfilled automatically as there $\chi_{i}=\mathcal{C}_{i} \cdot c_{1}$ was always 1 from isolatedness). One gains again the needed effectivity assertion.

b) In analogy to the reasoning in c) in app. B one gets here (for $n$ even; note $r>n$ ) that $h^{0}\left(\mathcal{E}, \mathcal{O}_{\mathcal{E}}\left(\frac{n}{2} s_{i}+\frac{r_{i}+\chi_{i}-2}{2} F\right)\right)=h^{0}\left(\mathcal{C}, \mathcal{O}_{\mathcal{C}}\left(\frac{r_{i}+\chi_{i}-2}{2}\right) \oplus \bigoplus_{j=2}^{n / 2} \mathcal{O}_{\mathcal{C}}\left(\frac{r_{i}+\chi_{i}-2}{2}-j\right)\right)=\frac{r_{i}+\chi_{i}-2}{2}+$ $1+\sum_{j=2}^{n / 2}\left(\frac{r_{i}+\chi_{i}-2}{2}-j+1\right)=\frac{n}{2}\left(\frac{r_{i}+\chi_{i}-2}{2}+1\right)-\left(\frac{1}{2} \frac{n}{2}\left(\frac{n}{2}+1\right)-1\right)$. So one gets for the multiplicity

$$
k \geq \begin{cases}1+\frac{n}{4}\left(r_{i}-\frac{n}{2}+\chi_{i}-1\right) & \text { for } n \equiv 0(2) \\ 1+r_{i}-\frac{(n-1) \chi_{i}+2}{2} & \text { for } n \neq 0(2)\end{cases}
$$

So b) of Theorem $P f a f f_{\text {all } C \subset X}$ in sect. 4.4 follows from (C.2): choosing $C$ ample in $Y$ makes $S-n \lambda$ ample in $X$, so $r_{i}>n \chi_{i} \geq n$ for $\lambda$ ample, giving again (3.11); the cases $n=3,4,5$ give $k \geq 2$ for all $\mathcal{C}_{i}$ from (C.2) (using $r_{i}-n \chi_{i}>0$ ). 


\section{References}

1. E. Witten, World-Sheet Corrections Via D-Instantons, hep-th/9907041, JHEP 0002 (2000) 030.

2. R. Friedman, J. Morgan and E. Witten, Vector Bundles and F-Theory, hepth/9701162, Comm. Math. Phys. 187 (1997) 679.

3. C. Beasley and E. Witten, Residues and World-Sheet Instantons, hep-th/0304115, JHEP 0310 (2003) 065.

4. E. Silverstein and E. Witten, "Criteria for Conformal Invariance of (0,2) Models", heo-th/9503212, Nucl.Phys. B444 (1995) 161.

5. D. Huybrechts, The Tangent Bundle of a Calabi-Yau Manifold - Deformations and restriction to Rational Curves, Comm. Math. Phys. 171 (1995) 139.

6. J. Distler, "Resurrecting (2,0) Compactifications", Phys.Lett. B188 (1987) 431;

J. Distler and B. Greene, "Aspects of (2,0) String Compactifications", Nucl.Phys. 304 (1988) 1.

7. E.I. Buchbinder, R. Donagi and B.A. Ovrut, Superpotentials for Vector Bundle Moduli, hep-th/0205190, Nucl.Phys. B653 (2003) 400; Vector Bundle Moduli Superpotentials in Heterotic Superstrings and M-Theory, hep-th/0206203, JHEP 0207 (2002) 066 .

8. E.I. Buchbinder and B.A. Ovrut, Vacuum Stability in Heterotic M-Theory, hepth/0310112, Phys.Rev. D69 (2004) 086010.

9. B.A. Ovrut, T. Pantev and J. Park, Small Instanton Transitions in Heterotic MTheory, hep-th/0001133, JHEP 0005 (2000) 045.

10. G. Curio, World-sheet Instanton Superpotentials in Heterotic String theory and their Moduli Dependence, arXiv:0810.3087, JHEP 0909 (2009) 125.

11. G. Curio, Perspectives on Pfaffians of Heterotic World-sheet Instantons, arXiv:0904.2738, JHEP 0909 (2009) 131.

12. G. Curio, Chiral matter and transitions in heterotic string models, hep-th/9803224, Phys.Lett. B435 (1998) 39.

13. G. Curio and R. Y. Donagi, Moduli in N=1 Heterotic/F-Theory Duality, hepth/9801057, Nucl.Phys. B518 (1998) 603. 